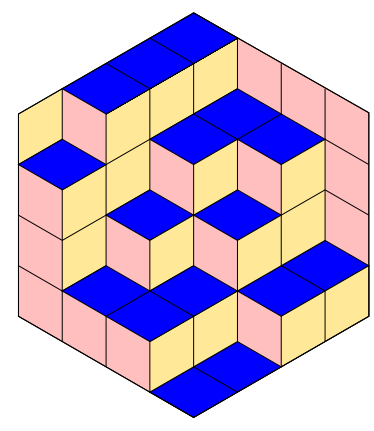

ALGEBRAIC COMBINATORICS

Mark S. MacLean \& Štefko Miklavič

Bipartite distance-regular graphs and taut pairs of pseudo primitive idempotents Volume 2, issue 4 (2019), p. 499-520.

<http://alco.centre-mersenne.org/item/ALCO_2019__2_4_499_0>

(C) The journal and the authors, 2019. Some rights reserved.

(c) BY This article is licensed under the

Creative Commons ATtribution 4.0 International LiCEnSE.

http://creativecommons.org/licenses/by/4.0/

Access to articles published by the journal Algebraic Combinatorics on the website http://alco.centre-mersenne.org/ implies agreement with the Terms of Use (http://alco.centre-mersenne.org/legal/).

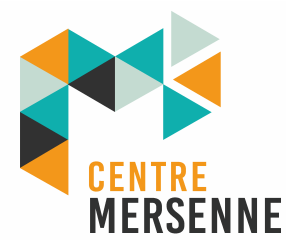

Algebraic Combinatorics is member of the Centre Mersenne for Open Scientific Publishing www.centre-mersenne.org 


\title{
Bipartite distance-regular graphs and taut pairs of pseudo primitive idempotents
}

\author{
Mark S. MacLean \& Štefko Miklavič
}

\begin{abstract}
Let $\Gamma$ denote a bipartite distance-regular graph with diameter $D \geqslant 4$, valency $k \geqslant 3$, and intersection numbers $c_{i}, b_{i}(0 \leqslant i \leqslant D)$. By a pseudo cosine sequence of $\Gamma$ we mean a sequence of complex scalars $\sigma_{0}, \sigma_{1}, \ldots, \sigma_{D}$ such that $\sigma_{0}=1$ and $c_{i} \sigma_{i-1}+b_{i} \sigma_{i+1}=k \sigma_{1} \sigma_{i}$ for $1 \leqslant i \leqslant D-1$. By an associated pseudo primitive idempotent of $\Gamma$, we mean a nonzero scalar multiple of the matrix $\sum_{i=0}^{D} \sigma_{i} A_{i}$, where $A_{0}, A_{1}, \ldots, A_{D}$ are the distance matrices of $\Gamma$. Given pseudo primitive idempotents $E, F$ of $\Gamma$, we define the pair $E, F$ to be taut whenever the entry-wise product $E \circ F$ is not a scalar multiple of a pseudo primitive idempotent, but is a linear combination of two pseudo primitive idempotents of $\Gamma$. In this paper, we determine all the taut pairs of pseudo primitive idempotents of $\Gamma$.
\end{abstract}

\section{INTRODUCTION}

Let $\Gamma$ denote a distance-regular graph with diameter $D \geqslant 4$ and Bose-Mesner algebra $M$. In [14], Terwilliger and Weng introduced the notion of a pseudo primitive idempotent. Given any complex number $\theta$, a nonzero matrix $E \in M$ is said to be a pseudo primitive idempotent of $\Gamma$ for $\theta$ whenever $(A-\theta I) E$ is a scalar multiple of $A_{D}$, where $A$ is the adjacency matrix and $A_{D}$ is the $D$ th distance matrix of $\Gamma$. We call $\theta$ the associated pseudo eigenvalue of $E$. By the associated pseudo cosine sequence, we mean a sequence of scalars $\sigma_{0}, \sigma_{1}, \ldots, \sigma_{D}$ such that $\sigma_{0}=1$ and

$$
c_{i} \sigma_{i-1}+a_{i} \sigma_{i}+b_{i} \sigma_{i+1}=\theta \sigma_{i} \quad(0 \leqslant i \leqslant D-1),
$$

where $\sigma_{-1}$ is indeterminate.

There have been a number of papers concerning pseudo primitive idempotents and their cosine sequences $[5,6,7,12,14]$. Given a pair of pseudo primitive idempotents $E, F$, in [12], Pascasio and Terwilliger defined the pair to be tight whenever the entrywise product $E \circ F$ is a scalar multiple of a single pseudo primitive idempotent. In their paper they determine all the tight pairs of pseudo primitive idempotents whose pseudo eigenvalues are real.

It is natural to consider the case where the entry-wise product $E \circ F$ is not a scalar multiple of a pseudo primitive idempotent, but instead is a linear combination of two pseudo primitive idempotents. We define such a pair $E, F$ to be taut. To make this

Manuscript received 8th May 2018, revised 1st November 2018, accepted 24th November 2018.

KEYWORDS. distance-regular graph, pseudo primitive idempotent, taut pair.

ACKNOWLEDGEMEnts. The second author acknowledges the financial support from the Slovenian Research Agency (research core funding No. P1-0285 and research projects N1-0038, N1-0062, J17051). 
problem manageable, we restrict our attention to bipartite distance-regular graphs. In [11], MacLean and Terwilliger posed the following two problems.

Problem 1.1. ([11, Problem 17.8]) Let $\Gamma$ denote a bipartite distance-regular graph with diameter $D \geqslant 4$. Find all the taut pairs of pseudo primitive idempotents of $\Gamma$.

Problem 1.2. ([11, Conjecture 17.9]) Let $\Gamma$ denote a bipartite distance-regular graph with diameter $D \geqslant 4$ and valency $k \geqslant 3$, and fix vertex $x$. Let $T$ denote the Terwilliger algebra of $\Gamma$ with respect to $x$. Assume that up to isomorphism there exist exactly two irreducible T-modules with endpoint 2, and they are both thin. Let E, F denote pseudo primitive idempotents associated with these modules. We conjecture the pair $E, F$ is taut.

For a bipartite distance-regular graph with valency $k$, a pseudo eigenvalue $\theta$ is said to be trivial whenever $\theta= \pm k$. A pseudo primitive idempotent or pseudo cosine sequence is said to be trivial whenever its associated pseudo eigenvalue is trivial. With this definition, we note Lang has already made some progress in attacking Problems 1.1, 1.2. Regarding Problem 1.1, see Theorem 5.4 for his main result. Regarding Problem 1.2, Lang proved in [7] that if $\Gamma$ is $Q$-polynomial with $D \geqslant 5$ and exactly two non-isomorphic irreducible $T$-modules with endpoint 2 , then the pair of pseudo primitive idempotents associated with the local eigenvalues of these modules do indeed form a taut pair. Lang also provides a feasible intersection array with diameter 4 for which the conjecture in Problem 1.2 is not satisfied. However, at this time no distance-regular graph is known to exist with this intersection array.

In this paper we solve Problem 1.1. Our results are organized as follows. Let $\Gamma$ denote a bipartite distance-regular graph with diameter $D \geqslant 4$ and valency $k \geqslant 3$, and define a parameter $\Delta_{2}$ in terms of the intersection numbers by

$$
\Delta_{2}=(k-2)\left(c_{3}-1\right)-\left(c_{2}-1\right) p_{22}^{2} .
$$

Let $E, F$ denote nontrivial pseudo primitive idempotents with associated pseudo eigenvalues $\theta, \theta^{\prime}$, respectively. We determine all taut pairs $E, F$ in the case $D=4$ in Theorem 4.9. Now assume $D \geqslant 5$. In Section 5 , we determine all taut pairs $E, F$ for which $\theta= \pm \theta^{\prime}$. Now assume $E, F$ is a taut pair for which $\theta \neq \pm \theta^{\prime}$. If one of $\theta, \theta^{\prime}$ is zero, this taut pair is described in Lemma 7.3. Now assume $\theta, \theta^{\prime}$ are both nonzero. If $\Delta_{2}=0$, this taut pair is described in Section 8. If $\Delta_{2} \neq 0$, this taut pair is described in Section 9.

We are very interested in seeing a solution to Problem 1.2 in the future, since we are interested in classifying the bipartite distance-regular graphs with exactly two irreducible $T$-modules of endpoint 2 , both of which are thin.

\section{Preliminaries}

In this section we review some definitions and basic results concerning distance-regular graphs. See the book of Brouwer, Cohen and Neumaier [2] for more background information.

Let $\mathbb{C}$ denote the complex number field and let $X$ denote a nonempty finite set. Let $\operatorname{Mat}_{X}(\mathbb{C})$ denote the $\mathbb{C}$-algebra consisting of all matrices whose rows and columns are indexed by $X$ and whose entries are in $\mathbb{C}$. Let $\Gamma=(X, \mathcal{R})$ denote a finite, undirected, connected graph, without loops or multiple edges, with vertex set $X$ and edge set $\mathcal{R}$. Let $\partial$ denote the path-length distance function for $\Gamma$, and set $D:=\max \{\partial(x, y) \mid x, y \in$ $X\}$. We call $D$ the diameter of $\Gamma$. For a vertex $x \in X$ and an integer $i$ let $\Gamma_{i}(x)$ denote the set of vertices at distance $i$ from $x$. We abbreviate $\Gamma(x):=\Gamma_{1}(x)$. For an integer $k \geqslant 0$ we say $\Gamma$ is regular with valency $k$ whenever $|\Gamma(x)|=k$ for all $x \in X$. We say $\Gamma$ 
is distance-regular whenever for all integers $h, i, j(0 \leqslant h, i, j \leqslant D)$ and for all vertices $x, y \in X$ with $\partial(x, y)=h$, the number

$$
p_{i j}^{h}:=\left|\Gamma_{i}(x) \cap \Gamma_{j}(y)\right|
$$

is independent of $x$ and $y$. The $p_{i j}^{h}$ are called the intersection numbers of $\Gamma$.

For the rest of this paper we assume $\Gamma$ is distance-regular with diameter $D \geqslant 4$ and valency $k \geqslant 3$. Note that $p_{i j}^{h}=p_{j i}^{h}$ for $0 \leqslant h, i, j \leqslant D$. For convenience set $c_{i}:=p_{1, i-1}^{i}(1 \leqslant i \leqslant D), a_{i}:=p_{1 i}^{i}(0 \leqslant i \leqslant D), b_{i}:=p_{1, i+1}^{i}(0 \leqslant i \leqslant D-1)$, $k_{i}:=p_{i i}^{0}(0 \leqslant i \leqslant D)$, and $c_{0}=b_{D}=0$. By the triangle inequality the following hold for $0 \leqslant h, i, j \leqslant D$ :

(i) $p_{i j}^{h}=0$ if one of $h, i, j$ is greater than the sum of the other two;

(ii) $p_{i j}^{h} \neq 0$ if one of $h, i, j$ equals the sum of the other two.

In particular $c_{i} \neq 0$ for $1 \leqslant i \leqslant D$ and $b_{i} \neq 0$ for $0 \leqslant i \leqslant D-1$. We observe that $\Gamma$ is regular with valency $k=k_{1}=b_{0}$ and that

$$
c_{i}+a_{i}+b_{i}=k \quad(0 \leqslant i \leqslant D) .
$$

Note that $k_{i}=\left|\Gamma_{i}(x)\right|$ for $x \in X$ and $0 \leqslant i \leqslant D$. By [2, p. 127],

$$
k_{i}=\frac{b_{0} b_{1} \cdots b_{i-1}}{c_{1} c_{2} \cdots c_{i}} \quad(0 \leqslant i \leqslant D) .
$$

We recall the Bose-Mesner algebra of $\Gamma$. For $0 \leqslant i \leqslant D$ let $A_{i}$ denote the matrix in $\operatorname{Mat}_{X}(\mathbb{C})$ with $(x, y)$-entry

$$
\left(A_{i}\right)_{x y}=\left\{\begin{array}{l}
1 \text { if } \partial(x, y)=i \\
0 \text { if } \partial(x, y) \neq i
\end{array} \quad(x, y \in X)\right.
$$

We call $A_{i}$ the $i$ th distance matrix of $\Gamma$. We abbreviate $A:=A_{1}$ and call this the adjacency matrix of $\Gamma$. We observe

(ai) $A_{0}=I$;

(aii) $\sum_{i=0}^{D} A_{i}=J$

(aiii) $\overline{A_{i}}=A_{i}(0 \leqslant i \leqslant D)$;

(aiv) $A_{i}^{t}=A_{i}(0 \leqslant i \leqslant D)$;

(av) $A_{i} A_{j}=\sum_{h=0}^{D} p_{i j}^{h} A_{h}(0 \leqslant i, j \leqslant D)$,

where $I$ (resp. $J$ ) denotes the identity matrix (resp. all 1's matrix) in $\operatorname{Mat}_{X}(\mathbb{C})$. Using these facts we find $A_{0}, A_{1}, \ldots, A_{D}$ is a basis for a commutative subalgebra $M$ of $\operatorname{Mat}_{X}(\mathbb{C})$. We call $M$ the Bose-Mesner algebra of $\Gamma$. It turns out that $A$ generates $M[1$, p. 190]. Since each entry in the distance matrices is either 0 or 1 , we have

$$
A_{i} \circ A_{j}=\delta_{i j} A_{i} \quad(0 \leqslant i, j \leqslant D),
$$

where $\circ$ denotes the entry-wise matrix product. It follows that $M$ is closed under $\circ$.

By [2, p. 45], $M$ has a second basis $E_{0}, E_{1}, \ldots, E_{D}$ such that

(ei) $E_{0}=|X|^{-1} J$;

(eii) $\sum_{i=0}^{D} E_{i}=I$;

(eiii) $\overline{E_{i}}=E_{i}(0 \leqslant i \leqslant D)$;

(eiv) $E_{i}^{t}=E_{i}(0 \leqslant i \leqslant D)$;

(ev) $E_{i} E_{j}=\delta_{i j} E_{i}(0 \leqslant i, j \leqslant D)$.

We call $E_{0}, E_{1}, \ldots, E_{D}$ the primitive idempotents of $\Gamma$. For $0 \leqslant i \leqslant D$ define scalars $\theta_{i}$ by

$$
A=\sum_{i=0}^{D} \theta_{i} E_{i} .
$$


Then $A E_{i}=E_{i} A=\theta_{i} E_{i}$ for $0 \leqslant i \leqslant D$, and $\theta_{0}=k$. The scalars $\theta_{0}, \theta_{1}, \ldots, \theta_{D}$ are real and distinct, since $A$ generates $M\left[1\right.$, p. 197]. The scalars $\theta_{0}, \theta_{1}, \ldots, \theta_{D}$ are known as the eigenvalues of $\Gamma$. We say that primitive idempotent $E_{i}$ is associated with the eigenvalue $\theta_{i}(0 \leqslant i \leqslant D)$. For each integer $i(0 \leqslant i \leqslant D)$, let $m_{i}$ denote the rank of $E_{i}$; we refer to $m_{i}$ as the multiplicity of $E_{i}$.

Let $E$ denote a primitive idempotent of $\Gamma$, and let $m$ denote the multiplicity of $E$. By $[1, \S I I .3]$, there exist real scalars $\sigma_{0}, \sigma_{1}, \ldots, \sigma_{D}$ such that $\sigma_{0}=1$ and

$$
E=|X|^{-1} m \sum_{i=0}^{D} \sigma_{i} A_{i} .
$$

The sequence $\sigma_{0}, \sigma_{1}, \ldots, \sigma_{D}$ is called the cosine sequence of $\Gamma$ associated with $E$. We abbreviate $\sigma:=\sigma_{1}$. By [2, Proposition 4.1.1], the following are equivalent:

(i) $\theta$ is an eigenvalue of $\Gamma$ and $\sigma_{0}, \sigma_{1}, \ldots, \sigma_{D}$ is the associated cosine sequence;

(ii) $\sigma_{0}=1$ and $c_{i} \sigma_{i-1}+a_{i} \sigma_{i}+b_{i} \sigma_{i+1}=\theta \sigma_{i} \quad(0 \leqslant i \leqslant D)$,

where $\sigma_{-1}$ and $\sigma_{D+1}$ are indeterminates.

Recall $\Gamma$ is bipartite whenever $a_{i}=0$ for $0 \leqslant i \leqslant D$. Setting $a_{i}=0$ in (2) we find

$$
b_{i}+c_{i}=k \quad(0 \leqslant i \leqslant D) .
$$

Assume $\Gamma$ is bipartite with diameter $D \geqslant 3$. By [2, Lemma 4.1.7], we have

$$
p_{2 i}^{i}=\frac{b_{i}\left(c_{i+1}-1\right)+c_{i}\left(b_{i-1}-1\right)}{c_{2}} \quad(1 \leqslant i \leqslant D-1) .
$$

Definition 2.1. Let $\Gamma$ denote a bipartite distance-regular graph with diameter $D \geqslant 4$ and valency $k \geqslant 3$. We define scalars $\Delta_{i}$ by

$$
\Delta_{i}=\left(b_{i-1}-1\right)\left(c_{i+1}-1\right)-\left(c_{2}-1\right) p_{2 i}^{i} \quad(2 \leqslant i \leqslant D-1) .
$$

$B y$ [3, Theorem 12], we have $\Delta_{i} \geqslant 0$ for $2 \leqslant i \leqslant D-1$. We note that $\Delta_{i}=0$ for $2 \leqslant i \leqslant D-2$ precisely when $\Gamma$ is almost 2-homogeneous in the sense of Curtin [4].

\section{Pseudo PRimitive idempotents and PSEudo Cosine Sequences}

Let $\Gamma$ denote a bipartite distance-regular graph with diameter $D \geqslant 4$ and valency $k \geqslant 3$. In this section we define pseudo eigenvalues and pseudo primitive idempotents of $\Gamma$ and review some of their properties.

DeFINITION 3.1. Let $\Gamma$ denote a bipartite distance-regular graph with diameter $D \geqslant 4$ and valency $k \geqslant 3$. Let $\theta$ denote any complex number; we call $\theta$ a pseudo eigenvalue. $B y$ the pseudo cosine sequence associated with $\theta$, we mean the sequence of complex numbers $\sigma_{0}, \sigma_{1}, \ldots, \sigma_{D}$ such that $\sigma_{0}=1$ and

$$
c_{i} \sigma_{i-1}+b_{i} \sigma_{i+1}=\theta \sigma_{i} \quad(0 \leqslant i \leqslant D-1),
$$

where $\sigma_{-1}$ is an indeterminate. We abbreviate $\sigma:=\sigma_{1}$. By a pseudo primitive idempotent associated with $\theta$, we mean a nonzero scalar multiple of the matrix

$$
\sum_{i=0}^{D} \sigma_{i} A_{i}
$$

With reference to Definition 3.1, we remark that $\theta \in \mathbb{R}$ is an eigenvalue of $\Gamma$ if and only if its associated pseudo cosine sequence satisfies $c_{D} \sigma_{D-1}=\theta \sigma_{D}$.

REMARK 3.2. We remark that in the literature about pseudo primitive idempotents also the case $\theta=\infty$ is often considered. We will not consider this case here, as this situation was studied in [7] (see [7, Lemma 4.1, Lemma 4.2]). 
LEMMA 3.3 ([7, Lemma 3.3]). Let $\Gamma$ denote a bipartite distance-regular graph with diameter $D \geqslant 4$. Let $\sigma_{0}, \sigma_{1}, \ldots, \sigma_{D}$ denote a pseudo cosine sequence of $\Gamma$ with associated pseudo eigenvalue $\theta$. Then for $0 \leqslant i \leqslant 4, \sigma_{i}=f_{i}(\theta)$, where

$$
\begin{gathered}
f_{0}(\lambda)=1, \quad f_{1}(\lambda)=\frac{\lambda}{k}, \quad f_{2}(\lambda)=\frac{\lambda^{2}-k}{k b_{1}}, \\
f_{3}(\lambda)=\frac{\lambda^{3}-\left(k+c_{2} b_{1}\right) \lambda}{k b_{1} b_{2}}, \quad f_{4}(\lambda)=\frac{\lambda^{4}-\left(k+c_{2} b_{1}+c_{3} b_{2}\right) \lambda^{2}+c_{3} k b_{2}}{k b_{1} b_{2} b_{3}} .
\end{gathered}
$$

LEMmA 3.4 (Christoffel-Darboux formula [1, Theorem III.1.3]). Let $\Gamma$ denote a distance-regular graph with diameter $D \geqslant 4$. Let $\sigma_{0}, \sigma_{1}, \ldots, \sigma_{D}$ and $\rho_{0}, \rho_{1}, \ldots, \rho_{D}$ denote pseudo cosine sequences of $\Gamma$. Then

$$
k(\sigma-\rho) \sum_{h=0}^{i} k_{h} \sigma_{h} \rho_{h}=k_{i} b_{i}\left(\sigma_{i+1} \rho_{i}-\sigma_{i} \rho_{i+1}\right) \quad(0 \leqslant i \leqslant D-1) .
$$

Proof. See [12, Lemma 3.2]. We note that the proof in [12] assumes each $\sigma_{i}, \rho_{i} \in \mathbb{R}$, but their proof also works when $\sigma_{i}, \rho_{i} \in \mathbb{C}$.

For bipartite distance-regular graphs, we now obtain some other formulas similar to the Christoffel-Darboux equations. We will need the following two corollaries.

COROllaRY 3.5 ([12, Lemmas 3.3, 3.4]). Let $\Gamma$ denote a bipartite distance-regular graph with diameter $D \geqslant 4$. Let $\sigma_{0}, \sigma_{1}, \ldots, \sigma_{D}$ denote complex numbers. Then the following (i)-(iv) are equivalent.

(i) $\sigma_{0}, \sigma_{1}, \ldots, \sigma_{D}$ is a pseudo cosine sequence.

(ii) $\sigma_{0}=1$ and

$$
c_{i}\left(\sigma_{i-1}-\sigma_{i+1}\right)=k\left(\sigma \sigma_{i}-\sigma_{i+1}\right) \quad(1 \leqslant i \leqslant D-1) .
$$

(iii) $\sigma_{0}=1$ and

$$
b_{i}\left(\sigma_{i+1}-\sigma_{i-1}\right)=k\left(\sigma \sigma_{i}-\sigma_{i-1}\right) \quad(1 \leqslant i \leqslant D-1) .
$$

(iv) $\sigma_{0}=1$ and

$$
k(\sigma-1) \sum_{h=0}^{i} k_{h} \sigma_{h}=k_{i} b_{i}\left(\sigma_{i+1}-\sigma_{i}\right) \quad(0 \leqslant i \leqslant D-1) .
$$

Proof. We note that the proof in [12] assumes each $\sigma_{i} \in \mathbb{R}$, but their proof also works for $\sigma_{i} \in \mathbb{C}$.

COROllaRY 3.6. Let $\Gamma$ denote a bipartite distance-regular graph with diameter $D \geqslant 4$, and let $\sigma_{0}, \sigma_{1}, \ldots, \sigma_{D}$ denote a pseudo cosine sequence of $\Gamma$. Then for any integer $i$ $(1 \leqslant i \leqslant D-1)$, the following are equivalent.

(i) $\sigma_{i+1}=\sigma_{i-1}$.

(ii) $\sigma \sigma_{i}=\sigma_{i-1}$.

(iii) $\sigma \sigma_{i}=\sigma_{i+1}$.

LEMMA 3.7. Let $\Gamma$ denote a bipartite distance-regular graph with diameter $D \geqslant 4$. Let $\sigma_{0}, \sigma_{1}, \ldots, \sigma_{D}$ and $\rho_{0}, \rho_{1}, \ldots, \rho_{D}$ denote pseudo cosine sequences of $\Gamma$. For $1 \leqslant i \leqslant$ D-1,

$$
k^{2}\left(\sigma^{2}-\rho^{2}\right) \sum_{\substack{0 \leqslant h \leqslant i-1 \\ i-h \text { odd }}} k_{h} \sigma_{h} \rho_{h}=k_{i} c_{i} b_{i}\left(\sigma_{i+1} \rho_{i-1}-\sigma_{i-1} \rho_{i+1}\right) .
$$

Proof. Similar to the proof of [8, Lemma 3.3]. 
COROLlaRY 3.8. Let $\Gamma$ denote a bipartite distance-regular graph with diameter $D \geqslant 4$. Let $\sigma_{0}, \sigma_{1}, \ldots, \sigma_{D}$ denote a pseudo cosine sequence of $\Gamma$. For $1 \leqslant i \leqslant D-1$,

$$
k^{2}\left(\sigma^{2}-1\right) \sum_{\substack{0 \leqslant h \leqslant i-1 \\ i-h \text { odd }}} k_{h} \sigma_{h}=k_{i} c_{i} b_{i}\left(\sigma_{i+1}-\sigma_{i-1}\right) .
$$

Proof. Similar to the proof of [8, Corollary 3.4].

\section{TIGHT PAIRS AND TAUT PAIRS OF PSEUdO PRIMITIVE IDEMPOTENTS}

Let $\Gamma$ denote a distance-regular graph with diameter $D \geqslant 4$ and valency $k \geqslant 3$. In this section we recall tight pairs and taut pairs of pseudo primitive idempotents of $\Gamma$.

DeFinition 4.1. Let $\Gamma$ denote a distance-regular graph with diameter $D \geqslant 4$ and valency $k \geqslant 3$. Let $E, F$ denote pseudo primitive idempotents for $\Gamma$. We say the pair $E, F$ is tight whenever the entry-wise product $E \circ F$ is a scalar multiple of a pseudo primitive idempotent of $\Gamma$. We say a pair of pseudo cosine sequences (or pseudo eigenvalues) is tight whenever its pair of associated pseudo primitive idempotents is tight.

In [12], Pascasio and Terwilliger determine all tight pairs of pseudo primitive idempotents whose pseudo eigenvalues are real. In the following definition we consider pairs of pseudo primitive idempotents whose entry-wise product is a linear combination of exactly two pseudo primitive idempotents.

Definition 4.2. Let $\Gamma$ denote a distance-regular graph with diameter $D \geqslant 4$ and valency $k \geqslant 3$. Let $E, F$ denote pseudo primitive idempotents for $\Gamma$. We say the pair $E, F$ is taut whenever the pair $E, F$ is not tight and the entry-wise product $E \circ F$ is a linear combination of two pseudo primitive idempotents of $\Gamma$. We say a pair of pseudo cosine sequences (or pseudo eigenvalues) is taut whenever its pair of associated pseudo primitive idempotents is taut. We note that if $E, F$ is taut, then any pair of nonzero scalar multiples of $E, F$ is taut.

We shall restrict our attention to bipartite distance-regular graphs. We first remind the reader of the possible tight pairs of pseudo primitive eigenvalues for such graphs.

ThEOREM 4.3 ([7, Lemmas 4.1, 4.2]). Let $\Gamma$ denote a bipartite distance-regular graph with diameter $D \geqslant 4$ and valency $k \geqslant 3$. Then (i)-(iii) hold below.

(i) The pair $\theta, k$ is tight for all $\theta \in \mathbb{C}$.

(ii) The pair $\theta,-k$ is tight for all $\theta \in \mathbb{C}$.

(iii) $\Gamma$ has no further tight pairs.

DeFINITION 4.4. Let $\Gamma$ denote a bipartite distance-regular graph with diameter $D \geqslant$ 4 and valency $k \geqslant 3$. Let $E$ denote a pseudo primitive idempotent with associated pseudo eigenvalue $\theta$ and pseudo cosine sequence $\sigma_{0}, \sigma_{1}, \ldots, \sigma_{D}$. We say $\theta$ is trivial whenever $\theta \in\{-k, k\}$. We note that $\theta$ is trivial if and only if $\sigma \in\{-1,1\}$. We say a pseudo primitive idempotent or pseudo cosine sequence is trivial whenever its associated pseudo eigenvalue is trivial.

Let $\Gamma$ denote a bipartite distance-regular graph with diameter $D \geqslant 4$ and valency $k \geqslant 3$. Let $E, F$ denote pseudo primitive idempotents with associated pseudo eigenvalues $\theta, \theta^{\prime}$. In the results that follow, we will typically assume $E, F$ are nontrivial in order to establish that the pair is not tight. Now assume the pair $E, F$ is taut. In this section we develop some equations involving $\theta, \theta^{\prime}$. First, we make a definition.

DEFINITION 4.5. Throughout Sections 4 and 5 , let $\Gamma$ denote a bipartite distance-regular graph with diameter $D \geqslant 4$ and valency $k \geqslant 3$. Let $\theta_{0}>\theta_{1}>\cdots>\theta_{D}$ denote the distinct eigenvalues of $\Gamma$, and for $0 \leqslant i \leqslant D$, let $E_{i}$ denote the primitive idempotent 
of $\Gamma$ associated with $\theta_{i}$. Furthermore, let $E$ and $F$ denote nontrivial pseudo primitive idempotents of $\Gamma$. We let $\theta, \theta^{\prime}$ denote the corresponding pseudo eigenvalues, and we let $\sigma_{0}, \sigma_{1}, \ldots, \sigma_{D}$ and $\rho_{0}, \rho_{1}, \ldots, \rho_{D}$ denote the corresponding pseudo cosine sequences. We let $G$ and $H$ denote pseudo primitive idempotents of $\Gamma$ with cosine sequences $\gamma_{0}, \gamma_{1}, \ldots, \gamma_{D}$ and $\epsilon_{0}, \epsilon_{1}, \ldots, \epsilon_{D}$, respectively, for which we assume $\gamma \neq \epsilon$.

LEMMA 4.6. With reference to Definition 4.5, the following (i), (ii) are equivalent.

(i) $E \circ F \in \operatorname{span}\{G, H\}$.

(ii) There exist complex scalars $a, b$ such that for $0 \leqslant i \leqslant D$,

$$
\sigma_{i} \rho_{i}=a \gamma_{i}+b \epsilon_{i}
$$

Suppose (i), (ii) hold. Then $a, b$ are nonzero and are given by

$$
a=\frac{\sigma \rho-\epsilon}{\gamma-\epsilon}, \quad b=\frac{\gamma-\sigma \rho}{\gamma-\epsilon} .
$$

Proof. Similar to the proof of [8, Lemma 4.2].

LEMma 4.7. With reference to Definition 4.5, assume conditions (i), (ii) hold in Lemma 4.6. Then $\gamma, \epsilon$ are both roots of the following polynomial in $\mathbb{C}[x]$ :

$$
(k-1) k^{2} b_{2} x^{2}-(k-1) k c_{2} \theta \theta^{\prime} x+\theta^{2} \theta^{\prime 2}\left(c_{2}-1\right)+\left(\theta^{2}+\theta^{\prime 2}-k^{2}\right) b_{2} .
$$

In particular,

$$
\gamma+\epsilon=\frac{c_{2} \theta \theta^{\prime}}{k b_{2}}, \quad \gamma \epsilon=\frac{\theta^{2} \theta^{\prime 2}\left(c_{2}-1\right)+\left(\theta^{2}+\theta^{\prime 2}-k^{2}\right) b_{2}}{(k-1) k^{2} b_{2}} .
$$

Proof. Without loss of generality, we show $\epsilon$ is a root of (16). We first observe $\sigma \rho \neq \epsilon$, since otherwise $a=0$, contradicting Lemma 4.6. Setting $i=2$ in (14) and eliminating $\sigma_{2}, \rho_{2}, \gamma_{2}, \epsilon_{2}$ using Lemma 3.3, we may solve the resulting equation for $\gamma$. Now setting $i=3$ in (14), applying Lemma 3.3, and replacing $\gamma$ in the result by the expression just obtained, we find $\epsilon$ is a root of (16), as desired.

ThEOREM 4.8. With reference to Definition 4.5, assume E, F is a taut pair. Then

(18) $b_{3}\left((k-2) b_{2}-\theta^{2}\left(c_{2}-1\right)\right)\left((k-2) b_{2}-\theta^{\prime 2}\left(c_{2}-1\right)\right)=-b_{1} \Delta_{2}\left(\theta^{2}-b_{2}\right)\left(\theta^{\prime 2}-b_{2}\right)$.

Proof. Observe conditions (i), (ii) hold in Lemma 4.6 for some pseudo primitive idempotents $G, H$. Using (15) and Lemma 3.3, one can show the expression

$$
(k-1)^{2} k^{2} b_{2}^{2} b_{3}^{2}\left(\sigma_{4} \rho_{4}-a \gamma_{4}-b \epsilon_{4}\right)
$$

is equal to the expression

$$
\begin{gathered}
(k-1) k b_{2} b_{3}\left(A\left((\gamma+\epsilon) \theta \theta^{\prime}-k^{2} \gamma \epsilon\right)-k^{2} \theta \theta^{\prime}(\gamma+\epsilon)\left((\gamma+\epsilon)^{2}-2 \gamma \epsilon\right)+k^{4} \gamma \epsilon\left((\gamma+\epsilon)^{2}-\gamma \epsilon\right)\right) \\
-k^{3} b_{2}^{2} c_{3}\left(b_{3}-1\right)-k b_{2} c_{3}\left(A\left(\theta^{2}+\theta^{\prime 2}\right)-\theta^{4}-\theta^{\prime 4}\right)+\theta^{2} \theta^{\prime 2}\left(A-\theta^{2}\right)\left(A-\theta^{\prime 2}\right),
\end{gathered}
$$

where $A=b_{2}+k c_{2}+c_{3} b_{2}$. Replacing $\gamma+\epsilon, \gamma \epsilon$ in this latter expression using (17), and then using (4), (5), one can show this latter expression equals $\left(k^{2}-\theta^{2}\right)\left(k^{2}-\right.$ $\left.\theta^{\prime 2}\right) c_{2}(k-1)^{-1} b_{2}^{-1}\left(b_{2}-1\right)^{-1}$ times the lefthand side of (18) minus the righthand side of (18). Since the expression in (19) equals 0 , the result follows.

With reference to Definition 4.5, our goal is to determine all the taut pairs of pseudo primitive idempotents of $\Gamma$. It turns out that the case $D=4$ is somewhat different than the case $D \geqslant 5$. Therefore, we treat this case separately. 
THEOREM 4.9. With reference to Definition 4.5 , assume that $D=4$. Then the following (i), (ii) are equivalent.

(i) $E, F$ is a taut pair.

(ii) $\theta, \theta^{\prime}$ satisfy (18) and the two roots of (16) are distinct.

\section{Proof.}

(i) $\Rightarrow$ (ii). If $E, F$ is a taut pair, then (18) holds by Theorem 4.8. Clearly, the roots of (16) are distinct in this case.

(ii) $\Rightarrow$ (i). Let $\gamma$ and $\epsilon$ be the roots of polynomial (16), and let $a, b$ be as in (15). Using (17), it is straightforward to show that (14) holds for $0 \leqslant i \leqslant 3$. It follows from the proof of Theorem 4.8 that since (18) holds we have also $\sigma_{4} \rho_{4}=a \gamma_{4}+b \epsilon_{4}$. The result follows.

With reference to Definition 4.5, for the rest of the paper we assume $D \geqslant 5$.

THEOREM 4.10. With reference to Definition 4.5, assume that $D \geqslant 5$ and $E, F$ is taut. Furthermore, assume $\theta, \theta^{\prime}$ are nonzero and that $\left(c_{3}-c_{2}\right)^{2}+\left(c_{4}-c_{3}\right)\left(c_{3}-c_{2}^{2}\right) \neq 0$. Then

$$
\begin{aligned}
\theta^{2} \theta^{\prime 2}= & \frac{b_{2}^{2}\left(-\left(c_{4}-1\right)\left(b_{2}+k c_{2}\right)+c_{3}^{2}\left(b_{4}-c_{2}\right)+c_{3}\left(c_{2} c_{4}+3 c_{4}+k c_{2}-2 k-2 c_{2}\right)\right)}{\left(c_{3}-c_{2}\right)^{2}+\left(c_{4}-c_{3}\right)\left(c_{3}-c_{2}^{2}\right)}, \\
\theta^{2}+\theta^{\prime 2}= & \frac{\left(b_{4}-c_{2}\right)\left(k-2 c_{2}+c_{2}^{2}\right) c_{3}^{2}-\left(c_{4}-1\right)\left(b_{2}^{2}+k c_{2}^{2}-c_{2}^{3}+k c_{2}^{3}\right)+c_{3} c_{2}^{3}\left(k+c_{4}-2\right)}{\left(c_{3}-c_{2}\right)^{2}+\left(c_{4}-c_{3}\right)\left(c_{3}-c_{2}^{2}\right)} \\
& +\frac{c_{3}\left(c_{2}^{2}\left(k c_{4}-k^{2}-k+3\right)+c_{2}\left(2 k^{2}-k-4 c_{4}-k c_{4}\right)-2 k\left(k-2 c_{4}\right)\right)}{\left(c_{3}-c_{2}\right)^{2}+\left(c_{4}-c_{3}\right)\left(c_{3}-c_{2}^{2}\right)} .
\end{aligned}
$$

Proof. Without loss of generality, we assume $G, H$ are the pseudo primitive idempotents satisfying the equivalent conditions (i), (ii) in Lemma 4.6. Setting $i=5$ in (14) and using Lemma 3.3, (6) and (3), we find

$$
\begin{gathered}
0=b_{1} b_{2} b_{3} b_{4}\left[-k^{4} \theta \theta^{\prime}\left(\left((\gamma+\epsilon)^{2}-2 \gamma \epsilon\right)^{2}-2(\gamma \epsilon)^{2}\right)+k^{6}(\gamma \epsilon)^{3}+k^{6} \gamma \epsilon(\gamma+\epsilon)\left((\gamma+\epsilon)^{2}-3 \gamma \epsilon\right)\right. \\
-k^{4} \theta \theta^{\prime} \gamma \epsilon\left((\gamma+\epsilon)^{2}-2 \gamma \epsilon\right)-k^{2} \gamma \epsilon\left(k^{4}\left((\gamma \epsilon)^{2}-\gamma \epsilon(\gamma+\epsilon)\right)+k^{2} \theta \theta^{\prime} \gamma \epsilon\right) \\
\left.+\left(c_{4} b_{3}+c_{3} b_{2}+c_{2} b_{1}+k\right)\left(k^{2} \theta \theta^{\prime}\left((\gamma+\epsilon)^{2}-2 \gamma \epsilon\right)-k^{2} \gamma \epsilon\left(k^{2}(\gamma+\epsilon)-\theta \theta^{\prime}\right)\right)\right]+C,
\end{gathered}
$$

where $C$ is the constant term with no factors of $\gamma, \epsilon$ and which is easily isolated using computer algebra software (but which is too lengthy to include here). Now using (17), once we divide by a factor of $\theta \theta^{\prime}\left(k^{2}-\theta^{2}\right)\left(k^{2}-\theta^{\prime 2}\right)$, we get a linear equation in the expressions $\theta^{2} \theta^{\prime 2}$ and $\theta^{2}+\theta^{\prime 2}$. Now multiplying out (18), we obtain another linear equation in the expressions $\theta^{2} \theta^{\prime 2}$ and $\theta^{2}+\theta^{\prime 2}$. Solving this linear system, we get the desired result.

LEMMA 4.11. With reference to Definition 4.5, assume that $D \geqslant 5$ and that the pair $\theta, \theta^{\prime}$ is taut. Furthermore, assume $\theta, \theta^{\prime}$ are nonzero. Then the following (i), (ii) are equivalent.

(i) $\Delta_{3}=\Delta_{2}=0$.

(ii) $\left(c_{3}-c_{2}\right)^{2}+\left(c_{4}-c_{3}\right)\left(c_{3}-c_{2}^{2}\right)=0$.

Proof.

(i) $\Rightarrow$ (ii). First, we show a couple of potential denominators are nonzero. Using $[2$, Proposition 4.1.6], we observe $\left(b_{2}-c_{3}\right)+c_{2}\left(c_{3}-c_{2}\right) \geqslant 0$. Suppose $\left(b_{2}-c_{3}\right)+c_{2}\left(c_{3}-c_{2}\right)=$ 0 . Then $b_{2}=c_{3}=c_{2}$, and so $c_{2}=k / 2$. Since $\Delta_{2}=0$, using (5) we find $k=2$, a contradiction. Hence $\left(b_{2}-c_{3}\right)+c_{2}\left(c_{3}-c_{2}\right)>0$. It's routine to show $k-3 c_{2}+c_{2}^{2}>0$. Now using (4) and (5), we may solve for $c_{3}, c_{4}$ in terms of $k, c_{2}$. It is then routine to check that (ii) holds. 
(ii) $\Rightarrow$ (i). Let $N$ denote the numerator of the expression for $\theta^{2} \theta^{\prime 2}$ in Theorem 4.10. By the proof of that theorem, since $\left(c_{3}-c_{2}\right)^{2}+\left(c_{4}-c_{3}\right)\left(c_{3}-c_{2}^{2}\right)=0$, we also have $N=0$. It's routine to show that

$$
b_{2}^{2}\left(c_{3}-1\right) c_{2} \Delta_{3}=(k-2) b_{2}^{2}\left(\left(c_{3}-c_{2}\right)^{2}+\left(c_{4}-c_{3}\right)\left(c_{3}-c_{2}^{2}\right)\right)-N\left(c_{2}-1\right) .
$$

It follows that either $\Delta_{3}=0$ or $c_{3}=1$. If $\Delta_{3}=0$, we are done by [9, Lemma 3.7]. Suppose $c_{3}=1$, so thus $c_{2}=1$. Using this information and the fact that $N=0$, we find $c_{4}=1$. Hence $\Delta_{3}=0$ by (5).

\section{The Case Where $\theta= \pm \theta^{\prime}$}

We say pseudo primitive idempotents $E, F$ are equivalent whenever $\theta=\theta^{\prime}$, where $\theta, \theta^{\prime}$ are the pseudo eigenvalues associated with $E, F$, respectively. In this case, $E, F$ are scalar multiples of each other. We say $E, F$ are opposites whenever $\theta=-\theta^{\prime}$. With reference to Definition 4.5, suppose for the moment that $E, F, G, H$ satisfy conditions (i), (ii) in Lemma 4.6. In this section, we consider the case when $E, F$ are equivalent or opposites. We will need the following lemmas.

Lemma 5.1. With reference to Definition 4.5, suppose E, $F$ are opposites. Then $\rho_{i}=$ $(-1)^{i} \sigma_{i}(0 \leqslant i \leqslant D)$.

Proof. Routine using (6) and induction.

LEMMA 5.2. With reference to Definition 4.5, for any pseudo primitive idempotent $K$, let $\hat{K}$ denote an opposite of $K$. Suppose $E \circ F \in \operatorname{span}\{G, H\}$. Then $\hat{E} \circ \hat{F} \in \operatorname{span}\{G, H\}$ and $E \circ \hat{F} \in \operatorname{span}\{\hat{G}, \hat{H}\}$.

Proof. Routine using Lemma 4.6 and Lemma 5.1.

In the following lemma, we recall this situation in the case when $E, F, G, H$ are actual primitive idempotents, not just pseudo primitive idempotents.

Lemma 5.3 ([8, Lemma 4.3]). With reference to Definition 4.5, suppose conditions (i), (ii) hold in Lemma 4.6, and suppose $E, F, G, H$ are primitive idempotents of $\Gamma$ (not just pseudo idempotents). Then

(i) $E, F$ are equivalent if and only if one of $G, H$ is equal to $E_{0}$.

(ii) $E, F$ are opposites if and only if one of $G, H$ is equal to $E_{D}$.

Furthermore, suppose $E=F$. Then $\Gamma$ is 2-homogeneous, and $E \in\left\{E_{1}, E_{D-1}\right\}$. Now suppose $E, F$ are opposites. Then $\Gamma$ is 2-homogeneous, and $E, F$ is a permutation of $E_{1}, E_{D-1}$.

In [6], Lang has worked out part of this case when $E, F$ are pseudo primitive idempotents.

TheOREM 5.4 ([6, Theorem 1.1]). With reference to Definition 4.5, the following (i), (ii) are equivalent.

(i) The entrywise product of $E$ with itself is a linear combination of $E_{0}$ and a pseudo primitive idempotent of $\Gamma$.

(ii) There exists a scalar $\lambda$ such that $\sigma_{i-1}+\lambda \sigma_{i}+\sigma_{i+1}=0$ for $1 \leqslant i \leqslant D-1$.

Moreover, $\Gamma$ has such a pseudo cosine sequence and pseudo primitive idempotent if and only if $\Gamma$ is almost 2-homogeneous with $c_{2} \geqslant 2$.

The following result will be useful.

LEMma 5.5. With reference to Definition 4.5, assume that $D \geqslant 5$. Furthermore, assume that conditions (i), (ii) hold in Lemma 4.6, and that E, F are either equivalent or opposites. Then $\Delta_{2}=0$. 
Proof. First, observe $\theta, \theta^{\prime}$ are nonzero, since if $\theta=\theta^{\prime}=0$, we quickly reach a contradiction using (18). Now observe that if $\left(c_{3}-c_{2}\right)^{2}+\left(c_{4}-c_{3}\right)\left(c_{3}-c_{2}^{2}\right)=0$, then $\Delta_{2}=0$ by Lemma 4.11 and we are done. Therefore, we assume $\left(c_{3}-c_{2}\right)^{2}+\left(c_{4}-c_{3}\right)\left(c_{3}-c_{2}^{2}\right) \neq 0$. Let $\theta=x+i y$ for some real numbers $x, y$ and let $\theta^{\prime}= \pm \theta$. Then we have that

$$
\theta^{2}+\theta^{\prime 2}=2\left(x^{2}-y^{2}\right)+4 i x y .
$$

Note that by Theorem 4.10 we have that (21) is real. It follows that either $x=0$ or $y=0$. Consequently, $\theta^{2}$ is real.

Setting $\theta^{\prime}= \pm \theta$ in (18), we find

$$
b_{3}\left((k-2) b_{2}-\theta^{2}\left(c_{2}-1\right)\right)^{2}=-b_{1} \Delta_{2}\left(\theta^{2}-b_{2}\right)^{2} .
$$

As $\theta^{2}$ is real, the expression on the left above is nonnegative, and the expression on the right is nonpositive. Hence both sides must equal 0 , and so we find $\Delta_{2}=0$.

Theorem 5.6. With reference to Definition 4.5 , assume that $D \geqslant 5$ and that conditions (i), (ii) hold in Lemma 4.6. Furthermore, assume E, $F$ are either equivalent or opposites. Then $c_{2}>1$, and $\theta^{2}=\theta^{\prime 2}=(k-2) b_{2}\left(c_{2}-1\right)^{-1}$. Furthermore, (i), (ii) hold below.

(i) $E, F$ are equivalent if and only if one of $G, H$ is a scalar multiple of $E_{0}$, and the other has associated pseudo eigenvalue $\left(k-2 c_{2}\right)\left(c_{2}-1\right)^{-1}$.

(ii) $E, F$ are opposites if and only if one of $G, H$ is a scalar multiple of $E_{D}$, and the other has associated pseudo eigenvalue $-\left(k-2 c_{2}\right)\left(c_{2}-1\right)^{-1}$.

Proof. Setting $\theta^{\prime}= \pm \theta$ in (18), we find

$$
b_{3}\left((k-2) b_{2}-\theta^{2}\left(c_{2}-1\right)\right)^{2}=-b_{1} \Delta_{2}\left(\theta^{2}-b_{2}\right)^{2} .
$$

As $\Delta_{2}=0$ by Lemma 5.5 , we have that $(k-2) b_{2}=\theta^{2}\left(c_{2}-1\right)$. Note that $c_{2}=1$ forces $k=2$, a contradiction. This shows that $\theta^{2}=\theta^{\prime 2}=(k-2) b_{2}\left(c_{2}-1\right)^{-1}$.

(i) Assume first that $E, F$ are equivalent. Setting $\theta^{\prime}=\theta= \pm \sqrt{(k-2) b_{2}\left(c_{2}-1\right)^{-1}}$ in (16) and factoring, we find $\{\gamma, \epsilon\}=\left\{1, \frac{k-2 c_{2}}{k\left(c_{2}-1\right)}\right\}$. The result follows. Without loss of generality, assume now that $G$ is a scalar multiple of $E_{0}$ and that $H$ has associated pseudo eigenvalue $\left(k-2 c_{2}\right)\left(c_{2}-1\right)^{-1}$. Note that $\epsilon=\left(k-2 c_{2}\right)\left(k\left(c_{2}-1\right)\right)^{-1}$, and so it follows from the equation on the left in (17) that $\theta \theta^{\prime}=(k-2) b_{2} /\left(c_{2}-1\right)^{-1}$. Plugging this and $x=\gamma=1$ into (16) we find

$$
\theta^{2}+\theta^{\prime 2}=\frac{2(k-2) b_{2}}{c_{2}-1}=2 \theta \theta^{\prime}
$$

It follows that $\theta=\theta^{\prime}$, and so $E, F$ are equivalent.

(ii) Similar to the proof of (i) above.

Corollary 5.7. With reference to Definition 4.5, assume that $D \geqslant 5$. Then $\Gamma$ has a taut pair of pseudo primitive idempotents that are equivalent or opposites if and only if $\Gamma$ is almost 2-homogeneous with $c_{2} \geqslant 2$.

Proof. Follows from Theorems 5.4, 5.6, 5.5 and Lemma 5.2.

\section{Equations involving Cosine SEquences}

With reference to Definition 4.5 , in the previous section we determined all taut pairs $E, F$ in which $E, F$ are equivalent or opposites. In the sections that follow we will determine all other taut pairs of primitive idempotents. To rule out this special case, we make the following definition. 
DeFINITION 6.1. Let $\Gamma$ denote a bipartite distance-regular graph with diameter $D \geqslant 5$ and valency $k \geqslant 3$. Let $E$ and $F$ denote nontrivial pseudo primitive idempotents of $\Gamma$ such that $E, F$ are neither equivalent nor opposites. We let $\theta, \theta^{\prime}$ denote the corresponding pseudo eigenvalues, and we let $\sigma_{0}, \sigma_{1}, \ldots, \sigma_{D}$ and $\rho_{0}, \rho_{1}, \ldots, \rho_{D}$ denote the corresponding pseudo cosine sequences.

LEMMA 6.2. With reference to Definition 6.1 , let $G, H$ denote nontrivial pseudo primitive idempotents with corresponding pseudo cosine sequences $\gamma_{0}, \gamma_{1}, \ldots, \gamma_{D}$ and $\epsilon_{0}, \epsilon_{1}, \ldots, \epsilon_{D}$, respectively. Fix an integer $i(0 \leqslant i \leqslant D-1)$ and suppose (14) holds for all nonnegative integers less than or equal to $i$, where $a, b$ are from (15). Then

$$
\frac{\sigma_{i+1} \rho_{i}-\sigma_{i} \rho_{i+1}}{\sigma-\rho}=a \frac{\gamma_{i+1}-\gamma_{i}}{\gamma-1}+b \frac{\epsilon_{i+1}-\epsilon_{i}}{\epsilon-1} .
$$

Proof. Similar to the proof of [8, Lemma 4.4], using (8), (11).

Lemma 6.3. With reference to Definition 6.1, let $G, H$ denote nontrivial pseudo primitive idempotents with corresponding pseudo cosine sequences $\gamma_{0}, \gamma_{1}, \ldots, \gamma_{D}$ and $\epsilon_{0}, \epsilon_{1}, \ldots, \epsilon_{D}$, respectively. Fix an integer $i(1 \leqslant i \leqslant D-1)$ and suppose (14) holds for all nonnegative integers less than $i$, where $a, b$ are from (15). Then

$$
\frac{\sigma_{i+1} \rho_{i-1}-\sigma_{i-1} \rho_{i+1}}{\sigma^{2}-\rho^{2}}=a \frac{\gamma_{i+1}-\gamma_{i-1}}{\gamma^{2}-1}+b \frac{\epsilon_{i+1}-\epsilon_{i-1}}{\epsilon^{2}-1}
$$

where $a, b$ are from (15).

Proof. Similar to the proof of [8, Lemma 4.5], using (12), (13).

With reference to Definition 6.1, in the rest of this section, we consider the equation

$$
\sigma_{i+1} \rho_{i+1}-\sigma_{i-1} \rho_{i-1}=\alpha \sigma_{i}\left(\rho_{i+1}-\rho_{i-1}\right)+\beta \rho_{i}\left(\sigma_{i+1}-\sigma_{i-1}\right),
$$

where

$$
\begin{aligned}
& \alpha=\frac{\theta}{k}+\frac{\theta\left(k^{2}-\theta^{2}\right)\left(b_{2}(k-2)-\theta^{\prime 2}\left(c_{2}-1\right)\right)}{k\left(\theta^{2}-\theta^{\prime 2}\right) b_{1} b_{2}}, \\
& \beta=\frac{\theta^{\prime}}{k}+\frac{\theta^{\prime}\left(k^{2}-\theta^{\prime 2}\right)\left(b_{2}(k-2)-\theta^{2}\left(c_{2}-1\right)\right)}{k\left(\theta^{\prime 2}-\theta^{2}\right) b_{1} b_{2}} .
\end{aligned}
$$

We shall see that this equation holds for $1 \leqslant i \leqslant D-1$ whenever $E \circ F$ is a linear combination of pseudo primitive idempotents $G, H$. We have a comment. Observe that if equation (24) holds for $1 \leqslant i \leqslant D-1$ for pair $E, F$, then it holds also for $1 \leqslant i \leqslant D-1$ for pair $E, \hat{F}$. To see this, change $\beta$ with $-\beta$ and use Lemma 5.1 . We now need two lemmas addressing the situation when one of $G, H$ is trivial.

Lemma 6.4. With reference to Definition 6.1 , assume (24) holds for $1 \leqslant i \leqslant D-1$. If 1 is a root of the polynomial in (16), then for $1 \leqslant i \leqslant D$,

$$
0=A\left(\sigma_{i} \rho_{i}+\sigma_{i-1} \rho_{i-1}\right)+B\left(\sigma_{i} \rho_{i-1}+\rho_{i} \sigma_{i-1}\right)+C,
$$

where

$$
\begin{aligned}
& A=(k-1) k^{2}\left(\theta+\theta^{\prime}\right) b_{2}, \\
& B=-\theta \theta^{\prime} k^{2}(k-1) c_{2}, \\
& C=-(k-1)\left(\theta+\theta^{\prime}\right)\left(k^{2} b_{2}+\theta \theta^{\prime}\left(b_{2}-k c_{2}\right)\right) .
\end{aligned}
$$

Proof. By our assumptions, we first note $Q=0$, where

$$
Q=(k-1) k^{2} b_{2}-(k-1) k c_{2} \theta \theta^{\prime}+\theta^{2} \theta^{\prime 2}\left(c_{2}-1\right)+\left(\theta^{2}+\theta^{\prime 2}-k^{2}\right) b_{2} .
$$


We proceed by induction on $i$. It is routine to show that equality holds in (27) for $i=1$. Now suppose $i \geqslant 2$ and that

$$
0=A\left(\sigma_{i-1} \rho_{i-1}+\sigma_{i-2} \rho_{i-2}\right)+B\left(\sigma_{i-1} \rho_{i-2}+\rho_{i-1} \sigma_{i-2}\right)+C .
$$

We will show equality holds in (27). Define scalars $\alpha, \beta$ as in (25), (26). Using (29), (24), and the fact that $Q=0$, we observe

$$
\begin{aligned}
& A\left(\sigma_{i} \rho_{i}+\right.\left.\sigma_{i-1} \rho_{i-1}\right)+B\left(\sigma_{i} \rho_{i-1}+\rho_{i} \sigma_{i-1}\right)+C \\
&= A\left(\sigma_{i} \rho_{i}+\sigma_{i-1} \rho_{i-1}\right)+B\left(\sigma_{i} \rho_{i-1}+\rho_{i} \sigma_{i-1}\right)+C \\
& \quad-\left(A\left(\sigma_{i-1} \rho_{i-1}+\sigma_{i-2} \rho_{i-2}\right)+B\left(\sigma_{i-1} \rho_{i-2}+\rho_{i-1} \sigma_{i-2}\right)+C\right) \\
&= A\left(\sigma_{i} \rho_{i}-\sigma_{i-2} \rho_{i-2}\right)+B\left(\sigma_{i-1}\left(\rho_{i}-\rho_{i-2}\right)+\rho_{i-1}\left(\sigma_{i}-\sigma_{i-2}\right)\right) \\
&= A\left(\alpha \sigma_{i-1}\left(\rho_{i}-\rho_{i-2}\right)+\beta \rho_{i-1}\left(\sigma_{i}-\sigma_{i-2}\right)\right) \\
& \quad \quad+B\left(\sigma_{i-1}\left(\rho_{i}-\rho_{i-2}\right)+\rho_{i-1}\left(\sigma_{i}-\sigma_{i-2}\right)\right) \\
&=(A \alpha+B) \sigma_{i-1}\left(\rho_{i}-\rho_{i-2}\right)+(A \beta+B) \rho_{i-1}\left(\sigma_{i}-\sigma_{i-2}\right) \\
&=\left(\frac{-k \theta Q}{\theta^{\prime}-\theta}\right) \sigma_{i-1}\left(\rho_{i}-\rho_{i-2}\right)+\left(\frac{k \theta^{\prime} Q}{\theta^{\prime}-\theta}\right) \rho_{i-1}\left(\sigma_{i}-\sigma_{i-2}\right) \\
&= 0 .
\end{aligned}
$$

Lemma 6.5. With reference to Definition 6.1 , assume 1 or -1 is a root of (16). Then the following (i), (ii) are equivalent.

(i) $E, F$ is a taut pair.

(ii) The polynomial in (16) has distinct roots, and there exist complex scalars $\alpha, \beta$ such that equality holds in (24) for all integers $i(1 \leqslant i \leqslant D-1)$.

Proof.

(i) $\Rightarrow$ (ii). Let $G, H$ denote pseudo primitive idempotents such that $E \circ F \in$ $\operatorname{span}\{G, H\}$, and let $\gamma_{0}, \gamma_{1}, \ldots, \gamma_{D}$ and $\epsilon_{0}, \epsilon_{1}, \ldots, \epsilon_{D}$ denote the pseudo cosine sequences associated with $G$ and $H$, respectively. Note that $\gamma \neq \epsilon$ (as $E, F$ is a taut pair) and that $\gamma, \epsilon$ are roots of (16).

First, assume 1 is a root of (16). Without loss of generality, we may assume $\epsilon=1$ and $\gamma=c_{2} \theta \theta^{\prime}\left(k b_{2}\right)^{-1}-1$. Thus $\epsilon_{i}=1(0 \leqslant i \leqslant D)$. Observe $Q=0$, where $Q$ is the expression in (28). We now proceed by induction. Let $\alpha, \beta$ be defined as in (25), (26). It's routine to check that (24) holds for $i=1$. Now suppose $i \geqslant 2$ and that (24) holds for all positive integers less than $i$. Following the proof and notation of Lemma 6.4, we find

$$
A\left(\sigma_{i} \rho_{i}+\sigma_{i-1} \rho_{i-1}\right)+B\left(\sigma_{i} \rho_{i-1}+\rho_{i} \sigma_{i-1}\right)+C=0 .
$$

By this fact and since $Q=0$, we find the expression

$A\left(\theta^{\prime}-\theta\right)\left(\sigma_{i} \rho_{i}+\sigma_{i-1} \rho_{i-1}\right)+\rho_{i} \sigma_{i-1}\left(B\left(\theta^{\prime}-\theta\right)-k \theta^{\prime} Q\right)+\sigma_{i} \rho_{i-1}\left(B\left(\theta^{\prime}-\theta\right)+k \theta Q\right)+\left(\theta^{\prime}-\theta\right) C$

is zero. By (9) and Lemma 4.6,

$$
\begin{aligned}
0 & =a\left(c_{i}\left(\gamma_{i-1}-\gamma_{i+1}\right)-k\left(\gamma \gamma_{i}-\gamma_{i+1}\right)\right) \\
& =c_{i}\left(\sigma_{i-1} \rho_{i-1}-\sigma_{i+1} \rho_{i+1}\right)-k\left(\gamma \sigma_{i} \rho_{i}-\sigma_{i+1} \rho_{i+1}-b(\gamma-1)\right),
\end{aligned}
$$

where $a, b$ are from (15). Now using (6) to eliminate $\sigma_{i+1}, \rho_{i+1}$ throughout, we find $k(k-1) b_{2}\left(\theta^{\prime 2}-\theta^{2}\right)$ times the expression in (33), minus the expression in (31), equals (34) $k(k-1) b_{2} b_{i}\left(\theta^{\prime 2}-\theta^{2}\right)\left(\sigma_{i+1} \rho_{i+1}-\sigma_{i-1} \rho_{i-1}-\alpha \sigma_{i}\left(\rho_{i+1}-\rho_{i-1}\right)-\beta \rho_{i}\left(\sigma_{i+1}-\sigma_{i-1}\right)\right)$.

Since the expressions in (33) and (31) are both zero, we find (24) holds at $i$, as desired. 
Next assume that -1 is a root of (16). By Lemma 5.2 the pair $E, \hat{F}$ is also taut, and corresponding $\hat{\epsilon}$ equals 1 . By the proof above, (24) holds for the pair $E, \hat{F}$. By the comment below (26), equation (24) holds also for the pair $E, F$.

(ii) $\Rightarrow$ (i). First, assume 1 is a root of (16). We define $\gamma, \epsilon$ to be the roots of the quadratic equation in (16), where $\epsilon=1$. Observe $\gamma=c_{2} \theta \theta^{\prime}\left(k b_{2}\right)^{-1}-1$ by (17). We define $\epsilon_{i}=1(0 \leqslant i \leqslant D)$ so that $\epsilon_{0}, \epsilon_{1}, \ldots, \epsilon_{D}$ is a trivial pseudo cosine sequence. Let $a, b$ be as defined in (15). We first show $a \neq 0$. Suppose to the contrary that $a=0$, so that $\sigma \rho=1$ and $\theta \theta^{\prime}=k^{2}$. Using (17), we routinely find $\theta^{2}+\theta^{\prime 2}=2 k^{2}$. Thus $\left(\theta-\theta^{\prime}\right)^{2}=\theta^{2}+\theta^{\prime 2}-2 \theta \theta^{\prime}=0$, so $\theta=\theta^{\prime}$, a contradiction. Hence $a \neq 0$. We now define $\gamma_{i}=\frac{\sigma_{i} \rho_{i}-b}{a}(0 \leqslant i \leqslant D)$. By Lemma 4.6 it suffices to show that $\gamma_{0}, \gamma_{1}, \ldots, \gamma_{D}$ is a pseudo cosine sequence, which we will do in view of Corollary 3.5 by showing $b_{i} \gamma_{i+1}-k \gamma \gamma_{i}+c_{i} \gamma_{i-1}=0$ for $1 \leqslant i \leqslant D-1$. We observe

(35) $a\left(b_{i} \gamma_{i+1}-k \gamma \gamma_{i}+c_{i} \gamma_{i-1}\right)=b_{i}\left(\sigma_{i+1} \rho_{i+1}-b\right)-k \gamma\left(\sigma_{i} \rho_{i}-b\right)+c_{i}\left(\sigma_{i-1} \rho_{i-1}-b\right)$.

We evaluate the right-hand side of the equation in (35) by first eliminating the product $\sigma_{i+1} \rho_{i+1}$ using (24), and then eliminating $\sigma_{i+1}, \rho_{i+1}$ in the result using (6). Recalling $\gamma=c_{2} \theta \theta^{\prime}\left(k b_{2}\right)^{-1}-1$ and using $(25),(26)$, we find the expression $k(k-1) b_{2}\left(\theta^{\prime 2}-\right.$ $\left.\theta^{2}\right) a\left(b_{i} \gamma_{i+1}-k \gamma \gamma_{i}+c_{i} \gamma_{i-1}\right)$ equals

$A\left(\theta^{\prime}-\theta\right)\left(\sigma_{i} \rho_{i}+\sigma_{i-1} \rho_{i-1}\right)+\rho_{i} \sigma_{i-1}\left(B\left(\theta^{\prime}-\theta\right)-k \theta^{\prime} Q\right)+\sigma_{i} \rho_{i-1}\left(B\left(\theta^{\prime}-\theta\right)+k \theta Q\right)+\left(\theta^{\prime}-\theta\right) C$,

where $Q$ is from (28). Since $Q=0$, the expression in (36) equals 0 by Lemma 6.4, and the result follows.

Assume now that -1 is a root of (16). Then 1 is a root of the equation obtained from (16) by changing $\theta^{\prime}$ with $-\theta^{\prime}$. By the proof above, the pair $E, \hat{F}$ is taut, and so by Lemma 5.2 also the pair $E, F$ is taut.

THEOREM 6.6. With reference to Definition 6.1, the following (i), (ii) are equivalent.

(i) $E, F$ is a taut pair.

(ii) The polynomial in (16) has distinct roots, and there exist complex scalars $\alpha, \beta$ such that equality holds in (24) for all integers $i \quad(1 \leqslant i \leqslant D-1)$.

Furthermore, suppose conditions (i), (ii) hold above. Then (25), (26) hold.

Proof.

(i) $\Rightarrow$ (ii). Let $G, H$ denote pseudo primitive idempotents such that $E \circ F \in$ $\operatorname{span}\{G, H\}$. Let $\gamma, \epsilon$ denote the first pseudo cosines of $G, H$, respectively. Note that $\gamma \neq \epsilon$, and by Lemma 4.7, $\gamma$ and $\epsilon$ are roots of (16). If $\gamma= \pm 1$ or $\epsilon= \pm 1$, then the result follows from Lemma 6.5. Suppose $\gamma \neq \pm 1$ and $\epsilon \neq \pm 1$. We first note that [8, Lemma 2.4] holds, since this proof works for pseudo primitive idempotents just as it does for primitive idempotents. The rest of our proof is similar to the proof of $[8$, Theorem 5.1], using [8, Lemma 2.4], (14), (22), (23).

(ii) $\Rightarrow$ (i). By assumption, for all $i(1 \leqslant i \leqslant D-1)$, we have

$$
\sigma_{i+1} \rho_{i+1}-\sigma_{i-1} \rho_{i-1}-\alpha \sigma_{i}\left(\rho_{i+1}-\rho_{i-1}\right)-\beta \rho_{i}\left(\sigma_{i+1}-\sigma_{i-1}\right)=0 .
$$

Define real numbers $\gamma, \epsilon$ to be the roots of the quadratic equation in (16). Now let $\gamma_{0}, \gamma_{1}, \ldots, \gamma_{D}$ and $\epsilon_{0}, \epsilon_{1}, \ldots, \epsilon_{D}$ denote the scalars satisfying $\gamma_{0}=\epsilon_{0}=1$ and

$$
c_{i} \gamma_{i-1}+b_{i} \gamma_{i+1}=k \gamma \gamma_{i}, \quad c_{i} \epsilon_{i-1}+b_{i} \epsilon_{i+1}=k \epsilon \epsilon_{i} \quad(1 \leqslant i \leqslant D-1) .
$$

Thus these sequences generated by $\gamma, \epsilon$ are pseudo cosine sequences by construction. If $\gamma= \pm 1$ or $\epsilon= \pm 1$, then the result follows from Lemma 6.5. So suppose $\gamma \neq \pm 1$, $\epsilon \neq \pm 1$. Define scalars $a, b$ as in (15). We will now show by induction on $j$ that

$$
\sigma_{j} \rho_{j}=a \gamma_{j}+b \epsilon_{j} \quad(0 \leqslant j \leqslant D) .
$$


It's routine to show that (39) holds for $i=0,1$. Now fix an integer $1 \leqslant i \leqslant D-1$ and assume (39) holds for all $j, 0 \leqslant j \leqslant i$. We shall show (39) holds for $j=i+1$. Adding (22) at $i$ to $(22)$ at $i-1$, we obtain

$$
\frac{\sigma_{i}\left(\rho_{i-1}-\rho_{i+1}\right)}{\sigma-\rho}-\frac{\rho_{i}\left(\sigma_{i-1}-\sigma_{i+1}\right)}{\sigma-\rho}=\frac{a\left(\gamma_{i+1}-\gamma_{i-1}\right)}{\gamma-1}+\frac{b\left(\epsilon_{i+1}-\epsilon_{i-1}\right)}{\epsilon-1} .
$$

Evaluating the left-hand side of (23) using [8, Lemma 2.4], we find

$$
\frac{\sigma \sigma_{i}\left(\rho_{i-1}-\rho_{i+1}\right)}{\sigma^{2}-\rho^{2}}-\frac{\rho \rho_{i}\left(\sigma_{i-1}-\sigma_{i+1}\right)}{\sigma^{2}-\rho^{2}}=\frac{a\left(\gamma_{i+1}-\gamma_{i-1}\right)}{\gamma^{2}-1}+\frac{b\left(\epsilon_{i+1}-\epsilon_{i-1}\right)}{\epsilon^{2}-1} .
$$

Now consider the equation $(*)$ which is $(37)$, plus $(\gamma+\epsilon)$ times the equation in $(40)$, minus the product of $(1+\gamma+\epsilon+\gamma \epsilon)$ with (41). In the equation $(*)$, the right-hand side is routinely shown to be equal to $a\left(\gamma_{i+1}-\gamma_{i-1}\right)+b\left(\epsilon_{i+1}-\epsilon_{i-1}\right)$. Now using $(25),(26)$, and (17), we find the left-hand side of the equation (*) equals $\sigma_{i+1} \rho_{i+1}-\sigma_{i-1} \rho_{i-1}$. By the inductive hypothesis, $\sigma_{i-1} \rho_{i-1}=a \gamma_{i-1}+b \epsilon_{i-1}$, and the result follows by Lemma 4.6.

Suppose now conditions (i), (ii) of the theorem hold. Solving (24) with $i=1,2$ for $\alpha$ and $\beta$ we routinely find that $\alpha, \beta$ are equal to the scalars (25), (26).

\section{WHEN CAN $\sigma_{i+1}=\sigma_{i-1}$ ?}

With reference to Definition 6.1, assume conditions (i), (ii) hold in Theorem 6.6. In Section 9 , we will obtain equations in which the expressions $\sigma \sigma_{i}-\sigma_{i-1}, \rho \rho_{i}-\rho_{i-1}$ appear as denominators. In this section we explore the circumstances under which these expressions can be zero. By Corollary 3.5, we note these expressions are zero precisely when $\sigma_{i+1}=\sigma_{i-1}$ and $\rho_{i+1}=\rho_{i-1}$. We will need the following two lemmas.

LEMMA 7.1. With reference to Definition 6.1 , at least one of $\sigma_{i-1}-\sigma_{i+1}, \sigma_{i}-\sigma_{i+2}$ is nonzero for $1 \leqslant i \leqslant D-2$.

Proof. Suppose, to the contrary, there exists an integer $i(1 \leqslant i \leqslant D-2)$ such that $\sigma_{i-1}-\sigma_{i+1}=0$ and $\sigma_{i}-\sigma_{i+2}=0$. Applying (9) at $i$ and $i+1$, we find

$$
\sigma \sigma_{i}=\sigma_{i+1} \quad \text { and } \quad \sigma \sigma_{i+1}=\sigma_{i+2} .
$$

Combining these two equations, we find $\sigma^{2} \sigma_{i}=\sigma_{i+2}=\sigma_{i}$. Suppose $\sigma_{i}=0$. Then by (42), $\sigma_{i+1}=0$. Using (6) and recursion, we find $\sigma_{0}=0$, a contradiction. Thus $\sigma_{i} \neq 0$ and hence $\sigma^{2}=1$. Thus $\theta$ is trivial, a contradiction.

Lemma 7.2. With reference to Definition 6.1, assume conditions (i), (ii) hold in Theorem 6.6. Then the following (i), (ii) are equivalent.

(i) $\alpha=\sigma$.

(ii) $b_{2}(k-2)-\theta^{\prime 2}\left(c_{2}-1\right)=0$ or $\theta=0$.

Moreover, suppose (i), (ii) hold above. Then

$$
\left(\sigma_{i+1}-\sigma_{i-1}\right)\left(\rho_{i+1}-(\beta+\rho) \rho_{i}+\rho_{i-1}\right)=0 \quad(1 \leqslant i \leqslant D-1) .
$$

Proof. The fact that (i), (ii) are equivalent follows from (25) and the fact that $\theta=k \sigma$. Now suppose (i), (ii) hold. Setting $\alpha=\sigma$ in (24), we find

(44) $\left(\sigma_{i+1}-\sigma \sigma_{i}\right) \rho_{i+1}-\left(\sigma_{i-1}-\sigma \sigma_{i}\right) \rho_{i-1}=\beta \rho_{i}\left(\sigma_{i+1}-\sigma_{i-1}\right) \quad(1 \leqslant i \leqslant D-1)$.

Now using (9), (10), we find that for $1 \leqslant i \leqslant D-1$,

$$
\sigma_{i+1}-\sigma \sigma_{i}=\frac{c_{i}}{k}\left(\sigma_{i+1}-\sigma_{i-1}\right), \quad \sigma_{i-1}-\sigma \sigma_{i}=\frac{b_{i}}{k}\left(\sigma_{i-1}-\sigma_{i+1}\right) .
$$

Evaluating (44) using (45), we find

$$
\left(\sigma_{i+1}-\sigma_{i-1}\right)\left(c_{i} \rho_{i+1}-\beta k \rho_{i}+b_{i} \rho_{i-1}\right)=0 .
$$


Using (6), it's routine to show $c_{i} \rho_{i+1}-\beta k \rho_{i}+b_{i} \rho_{i-1}=k\left(\rho_{i+1}-(\beta+\rho) \rho_{i}+\rho_{i-1}\right)$, and the result follows.

With reference to Definition 6.1, in the following lemma we describe the taut pairs $\theta, \theta^{\prime}$ for which $\theta=0$. We note this case is one instance in which $\sigma_{i+1}=\sigma_{i-1}$. In particular, $\sigma_{i+1}=\sigma_{i-1}=0$ for even integers $i(1 \leqslant i \leqslant D-1)$.

LEMMA 7.3. With reference to Definition 6.1 , assume $\theta=0$. Then the following (i), (ii) are equivalent.

(i) $\theta, \theta^{\prime}$ is a taut pair.

(ii) $\exists \beta \in \mathbb{C}$ such that $\rho_{i+1}-(\beta+\rho) \rho_{i}+\rho_{i-1}=0$ for $i$ odd, $1 \leqslant i \leqslant D-1$.

Assume (i), (i) hold above, and let $G, H$ denote pseudo primitive idempotents such that $E \circ F \in \operatorname{span}\{G, H\}$. Then $c_{3}>1, \theta^{\prime 2}=\frac{c_{2}\left(b_{3}-1\right)(k-1)}{c_{3}-1}+k$, and $G, H$ are opposites. Furthermore, $\beta$ is given by (26).

Proof. Using (6), we routinely find

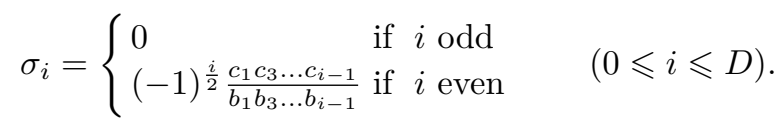

(i) $\Rightarrow$ (ii). By Lemma 7.2, (43) holds. The result now follows from (47).

(ii) $\Rightarrow$ (i). Define $\alpha:=0$. Using (47) and the assumption, it's routine to show (24) holds for $1 \leqslant i \leqslant D-1$. Since $\theta=0$ and $\theta^{\prime}$ is nontrivial, it is immediate that the polynomial in (16) has distinct roots. The result now follows from Theorem 6.6.

Now assume (i), (ii) hold, and let $G, H$ denote pseudo primitive idempotents such that $E \circ F \in \operatorname{span}\{G, H\}$. First, it follows from Theorem 6.6 that $\beta$ is given by (26). Now suppose $c_{3}=1$. Then $c_{2}=1$, and hence $\Delta_{2}=0$ by (5). Thus $k=2$ by (18), a contradiction. Hence $c_{3}>1$. The remaining results are routinely found using (18), (5), and (17).

With reference to Definition 6.1 assume conditions (i), (ii) hold in Theorem 6.6, and that $\alpha=\sigma$. If $\theta \neq 0$, then we have $b_{2}(k-2)-\theta^{\prime 2}\left(c_{2}-1\right)=0$ by Lemma 7.2. We will show that this implies $\Delta_{2}=0$, and this case will be further studied in Section 8 .

Lemma 7.4. With reference to Definition 6.1, assume conditions (i), (ii) hold in Theorem 6.6. Furthermore, assume $\alpha \neq \sigma, \beta \neq \rho$. Suppose $\sigma_{i+1}=\sigma_{i-1}$ or $\rho_{i+1}=\rho_{i-1}$ for some $i, 1 \leqslant i \leqslant D-1$. Then $b_{i-1}=1$ and $c_{2}=1$. In particular, $\Delta_{i}=0$, and $i>\frac{D}{2}+1$.

Proof. Let $i$ denote the minimal integer $(1 \leqslant i \leqslant D-1)$ such that $\sigma_{i+1}=\sigma_{i-1}$ or $\rho_{i+1}=\rho_{i-1}$. Without loss of generality, assume $\sigma_{i+1}=\sigma_{i-1}$. It's routine to show $\sigma_{2} \neq \sigma_{0}$, so $i \geqslant 2$. Thus $\sigma_{i} \neq \sigma_{i-2}$. By Corollary 3.6, $\sigma \sigma_{i}=\sigma_{i-1}=\sigma_{i+1}$. Setting $\sigma_{i+1}=\sigma \sigma_{i}$ and $\sigma_{i-1}=\sigma \sigma_{i}$ in (24) and simplifying, we find

$$
\sigma_{i}(\sigma-\alpha)\left(\rho_{i+1}-\rho_{i-1}\right)=0 .
$$

If $\sigma_{i}=0$, then using (6) we find $\sigma_{i}=\sigma_{i-2}=0$, a contradiction. Thus $\rho_{i+1}=\rho_{i-1}$, and $\rho_{i} \neq \rho_{i-2}$. Replacing $i$ by $i-1$ in (9) and recalling $\sigma_{i-1}=\sigma \sigma_{i}, \rho_{i-1}=\rho \rho_{i}$, we find

$$
\begin{aligned}
& c_{i-1}\left(\sigma_{i-2}-\sigma_{i}\right)=k \sigma_{i}\left(\sigma^{2}-1\right), \\
& c_{i-1}\left(\rho_{i-2}-\rho_{i}\right)=k \rho_{i}\left(\rho^{2}-1\right) .
\end{aligned}
$$

Combining these two equations, we find

$$
\left(\sigma_{i-2}-\sigma_{i}\right)\left(\rho^{2}-1\right) \rho_{i}-\left(\rho_{i-2}-\rho_{i}\right)\left(\sigma^{2}-1\right) \sigma_{i}=0 .
$$


Replacing $i$ by $i-1$ in (24) and using the facts that $\sigma_{i-1}=\sigma \sigma_{i}, \rho_{i-1}=\rho \rho_{i}$, we find

$$
\sigma_{i} \rho_{i}-\sigma_{i-2} \rho_{i-2}-\alpha \sigma \sigma_{i}\left(\rho_{i}-\rho_{i-2}\right)-\beta \rho \rho_{i}\left(\sigma_{i}-\sigma_{i-2}\right)=0 .
$$

Adding $\alpha \sigma \sigma_{i}-\sigma_{i-2}$ times (51) and $\left(\sigma^{2}-1\right) \sigma_{i}$ times (52) and simplifying using $(25),(26)$, we find $\sigma_{i-2}=\sigma_{i}\left(k \sigma^{2}-1\right)(k-1)^{-1}$. Plugging this into (49), we find $c_{i-1}=k-1$. Thus $b_{i-1}=1$. Now since $\Delta_{i} \geqslant 0$, using (5) we find $\Delta_{i}=0$ and $c_{2}=1$. The final remark follows from [2, Proposition 4.1.6(ii)].

COROllary 7.5. With reference to Definition 6.1, assume conditions (i), (ii) hold in Theorem 6.6. Furthermore, assume $\Delta_{2} \neq 0$, and $\theta, \theta^{\prime}$ are both nonzero. Then $\alpha \neq \sigma, \beta \neq \rho, \sigma_{i+1} \neq \sigma_{i-1}, \rho_{i+1} \neq \rho_{i-1}(1 \leqslant i \leqslant D-2)$. If in addition $\Delta_{D-1} \neq 0$, then also $\sigma_{D} \neq \sigma_{D-2}, \rho_{D} \neq \rho_{D-2}$.

Proof. Suppose $\alpha=\sigma$. Then by Lemma $7.2, b_{2}(k-2)=\left(c_{2}-1\right) \theta^{\prime 2}$. Now by (18), we find $\theta^{2}=b_{2}$. Setting $i=4$ in (24) and using (5), we routinely find $\Delta_{3}=0$. But then also $\Delta_{2}=0$ by [9, Lemma 3.7], a contradiction. Hence $\alpha \neq \sigma$, and similarly, $\beta \neq \rho$. Assume now that $\sigma_{i+1}=\sigma_{i-1}$ or $\rho_{i+1}=\rho_{i-1}$ for some $i(1 \leqslant i \leqslant D-2)$. By Lemma 7.4 we have $b_{i-1}=1$ and $c_{2}=1$. As $3+(i-1) \leqslant 3+D-3=D$, by [2, Proposition 4.1.6(ii)] we also have that $c_{3} \leqslant b_{i-1}$, implying $c_{3}=1$. But this forces $\Delta_{2}=0$, a contradiction. If $\Delta_{D-1} \neq 0$, then we have $\sigma_{D} \neq \sigma_{D-2}, \rho_{D} \neq \rho_{D-2}$ by Lemma 7.4.

\section{THE CASE $\Delta_{2}=0$}

With reference to Definition 6.1 , in this section we consider the case when $\Delta_{2}=0$. We will need the following lemma.

Lemma 8.1. With reference to Definition 6.1 , assume $\Delta_{2}=0$. Then $D \leqslant 5$ or $c_{2} \leqslant 2$. Moreover, if $\Gamma$ has a taut pair of pseudo eigenvalues, then $c_{2}>1$.

Proof. By [10, Theorem 4.4], $D \leqslant 5$ or $c_{2} \leqslant 2$. If $\Gamma$ has a taut pair of pseudo eigenvalues, then we observe $c_{2} \neq 1$ by Theorem 4.8 .

We now consider the case when $\Delta_{i}=0(2 \leqslant i \leqslant D-2)$. We note that this is precisely the case when $\Gamma$ is almost 2 -homogeneous.

LEMMA 8.2. With reference to Definition 6.1 , assume $c_{2}>1$ and $\Delta_{i}=0(2 \leqslant i \leqslant$ $D-2)$. Assume $\theta^{\prime}$ satisfies $(k-2) b_{2}=\left(c_{2}-1\right) \theta^{\prime 2}$. Then the following (i)-(iii) hold.

(i) If $c_{2}=2$, then the pair $\theta, \theta^{\prime}$ is taut for all $\theta \in \mathbb{C}$.

(ii) If $c_{2}>2$, then $D=5$ and the pair $\theta, \theta^{\prime}$ is taut for all $\theta \in \mathbb{C}$ 、 $\left\{ \pm \sqrt{-4 b_{2} /\left(c_{2}-2\right)}\right\}$.

(iii) $\Gamma$ has no other taut pairs.

Proof. (i) Using (5) and induction, we routinely find $c_{i}=i(1 \leqslant i \leqslant D-1)$. Observe $\theta^{\prime 2}=(k-2)^{2}$. We prove the result for $\theta^{\prime}=k-2$; the result for $\theta^{\prime}=-(k-2)$ then follows from Lemma 5.2. Let $\theta \in \mathbb{C}$ and let $\sigma_{0}, \sigma_{1}, \ldots, \sigma_{D}$ and $\rho_{0}, \rho_{1}, \ldots, \rho_{D}$ denote the pseudo cosines sequences for $\theta$ and $\theta^{\prime}$, respectively. Using (6) and induction, we routinely find $\rho_{i}=(k-2 i) / k(0 \leqslant i \leqslant D)$. Setting $\alpha=\sigma$ and $\beta=2-\rho$ and using (6), we find (24) holds for $1 \leqslant i \leqslant D-1$. Moreover, it's routine to show the polynomial in (16) has distinct roots in this case. Hence $\theta, \theta^{\prime}$ is a taut pair by Theorem 6.6 .

(ii) First, observe $D=5$ by Lemma 8.1. Using (5), we may solve for $c_{3}, c_{4}$ in terms of $k, c_{2}$. Now using Lemma 3.3, (6), (25), and (26), it's routine to check that (24) holds for $1 \leqslant i \leqslant D-1$. Using the fact that $\theta^{2} \neq-4 b_{2} /\left(c_{2}-2\right)$, it's routine to show the polynomial in (16) has distinct roots. Hence $\theta, \theta^{\prime}$ is a taut pair by Theorem 6.6. 
(iii) Let $\theta, \Psi \in \mathbb{C}$, and assume this is a taut pair of pseudo eigenvalues. Setting $\Delta_{2}=0$ in (18), we find at least one of $\theta^{2}, \Psi^{2}$ is equal to $(k-2) b_{2} /\left(c_{2}-1\right)$, and the result follows.

In the rest of this section, we consider the case when $\Delta_{2}=0$ and there exists an integer $i(3 \leqslant i \leqslant D-2)$ for which $\Delta_{i} \neq 0$. With reference to Lemma 8.1, it is natural to treat cases $D=5$ and $c_{2}=2$ separately. We shall classify the taut pairs of pseudo primitive idempotents in each case.

Lemma 8.3. With reference to Definition 6.1 , assume $D=5$. Furthermore, assume $\Delta_{2}=0$ and $\Delta_{3} \neq 0$. Then the following (i), (ii) are equivalent.

(i) $\theta, \theta^{\prime}$ is a taut pair.

(ii) $\left\{\theta^{2}, \theta^{\prime 2}\right\}=\left\{0, \frac{b_{2}(k-2)}{c_{2}-1}\right\}$ or $\left\{\theta^{2}, \theta^{\prime 2}\right\}=\left\{b_{2}+c_{2} b_{3}, \frac{b_{2}(k-2)}{c_{2}-1}\right\}$.

Proof. First, observe that since $\Delta_{2}=0$, we find using (5) that

$$
c_{3}=\frac{b_{2}-k c_{2}-c_{2}^{2}+k c_{2}^{2}}{k-3 c_{2}+c_{2}^{2}} .
$$

(i) $\Rightarrow$ (ii). Since $\Delta_{2}=0$ and regarding (18), without loss of generality, we may assume $b_{2}(k-2)-\theta^{\prime 2}\left(c_{2}-1\right)=0$. Thus by $(26)$ we find

$$
\beta=\frac{\theta^{\prime}\left(k c_{2}-b_{2}\right)}{k b_{2}} .
$$

Observe (43) holds by Lemma 7.2. Using the above information and Lemma 3.3, we may verify that $\rho_{i+1}-(\beta+\rho) \rho_{i}+\rho_{i-1}=0$ for $i=1,2,3$, and cannot be zero for $i=4$, or else $\Gamma$ is almost 2-homogeneous by Theorem 5.4 . Thus by (43), $\sigma_{5}=\sigma_{3}$. Using (6) and (53), we routinely find that $\sigma_{5}=\sigma_{3}$ implies either $\theta=0$ or $\theta^{2}=b_{2}+c_{2} b_{3}$. The result follows.

(ii) $\Rightarrow\left(\right.$ i). Without loss of generality, assume $b_{2}(k-2)-\theta^{\prime 2}\left(c_{2}-1\right)=0$. Let

$$
\alpha=\sigma, \quad \beta=\frac{\theta^{\prime}\left(k c_{2}-b_{2}\right)}{k b_{2}} .
$$

Using Lemma 3.3 and (6), it's now routine to show (24) holds for each $i, 1 \leqslant i \leqslant 4$. It's also routine to show the polynomial in (16) has distinct roots. The result now follows from Theorem 6.6.

LEMMA 8.4. With reference to Definition 6.1 , assume $b_{2}(k-2)=\left(c_{2}-1\right) \theta^{\prime 2}$, and that there exists an integer $i(2 \leqslant i \leqslant D-2)$ such that

$$
\rho_{i}-(\beta+\rho) \rho_{i-1}+\rho_{i-2}=0, \quad \rho_{i+2}-(\beta+\rho) \rho_{i+1}+\rho_{i}=0,
$$

where $\beta$ is from (26). Then $\Delta_{i}=0$.

Proof. Applying (9) at $i-1$ and $i+1$, we find

$$
c_{i-1}\left(\rho_{i-2}-\rho_{i}\right)=k\left(\rho \rho_{i-1}-\rho_{i}\right), \quad c_{i+1}\left(\rho_{i}-\rho_{i+2}\right)=k\left(\rho \rho_{i+1}-\rho_{i+2}\right) .
$$

Now solving (56) for $\rho_{i-2}, \rho_{i+2}$ and substituting into (57), we find

$$
\begin{aligned}
\left((\beta+\rho) c_{i-1}-k \rho\right) \rho_{i-1}+\left(k-2 c_{i-1}\right) \rho_{i} & =0, \\
\left(-k+2 c_{i+1}\right) \rho_{i}+\left(b_{i+1}(\beta+\rho)-k \rho\right) \rho_{i+1} & =0 .
\end{aligned}
$$

Furthermore, by (6),

$$
c_{i} \rho_{i-1}-k \rho \rho_{i}+b_{i} \rho_{i+1}=0 .
$$

We observe that we have three linear equations in the variables $\rho_{i-1}, \rho_{i}, \rho_{i+1}$. This system has nontrivial solutions, so the associated determinant must be zero. Setting this determinant equal to zero and using (5), (26), and the fact that $\theta^{\prime 2}=b_{2}(k-$ $2)\left(c_{2}-1\right)^{-1}$, we find $\Delta_{i}=0$, as desired. 
Recall that we are assuming that there exists an integer $i(3 \leqslant i \leqslant D-2)$ for which $\Delta_{i} \neq 0$. Define a positive number $f$ by

$$
f=\min \left\{i \geqslant 3 \mid \Delta_{i} \neq 0\right\}
$$

and note that $f \leqslant D-2$.

THEOREM 8.5. With reference to Definition 6.1, assume that $\theta, \theta^{\prime}$ is a taut pair, and that $\Delta_{2}=0, c_{2}=2$. Then the following (i)- (iv) hold.

(i) One of $\theta, \theta^{\prime}$ is equal to $\pm(k-2)$.

(ii) $f=D-2$, where $f$ is as defined above.

(iii) $c_{i}=i$ for $1 \leqslant i \leqslant D-2$.

(iv) Assume $\theta^{\prime}\left(\theta\right.$, respectively) is equal to $\pm(k-2)$. Then $\sigma_{D}=\sigma_{D-2}\left(\rho_{D}=\rho_{D-2}\right.$, respectively).

Proof. Observe that by (18), we have that either $\theta^{2}$ or $\theta^{\prime 2}$ is equal to $(k-2) b_{2} /\left(c_{2}-1\right)$. As $c_{2}=2$ and $b_{2}=k-2$, this shows part (i) of the theorem.

For the rest of the proof, assume without loss of generality that $\theta^{\prime 2}=(k-2)^{2}$. By [13, Lemma 3.6(ii)], we have $c_{i}=i$ for $1 \leqslant i \leqslant f$. Using (6), it is now straightforward to show that for $0 \leqslant i \leqslant f+1$ we have that either $\rho_{i}=(k-2 i) / k$ (if $\theta^{\prime}=k-2$ ), or $\rho_{i}=(-1)^{i}(k-2 i) / k$ (if $\theta^{\prime}=-(k-2)$ ). Consider now equation (43) from Lemma 7.2. Clearly $\sigma_{2}-\sigma_{0} \neq 0$, and so $\rho_{2}-(\beta+\rho) \rho_{1}+\rho_{0}=0$ implies that $\beta+\rho$ is either 2 (if $\left.\theta^{\prime}=k-2\right)$, or -2 (if $\theta^{\prime}=-(k-2)$ ). It follows that $\rho_{i+1}-(\beta+\rho) \rho_{i}+\rho_{i-1}=0$ for $1 \leqslant i \leqslant f$. Consider now $\rho_{f+2}-(\beta+\rho) \rho_{f+1}+\rho_{f}$. If this expression is equal to 0 , then $\Delta_{f}=0$ by Lemma 8.4, a contradiction. Therefore, $\sigma_{f+2}-\sigma_{f}=0$ by (43).

Note that if $D=5$, we have $f=D-2$ by our assumptions, so consider now the case $D \geqslant 6$. Assume that $f \leqslant D-3$. Then $\sigma_{f+3}-\sigma_{f+1} \neq 0$ by Lemma 7.1 , and so $\rho_{f+3}-(\beta+\rho) \rho_{f+2}+\rho_{f+1}=0$ by (43). By Lemma 8.4 we have that $\Delta_{f+1}=0$, contradicting [13, Lemma 4.13(iv)]. This shows that $f=D-2$. Claims (iii) and (iv) of the theorem now follow from the comments above.

THEOREM 8.6. With reference to Definition 6.1 , assume $\Delta_{2}=0, c_{2}=2$, and $\Delta_{D-2} \neq$ 0 . Then the following (i), (ii) are equivalent.

(i) The pair $\theta, \theta^{\prime}$ is taut.

(ii) $c_{i}=i(1 \leqslant i \leqslant D-2)$, and either $\theta^{\prime}= \pm(k-2)$ and $\sigma_{D}=\sigma_{D-2}$, or $\theta= \pm(k-2)$ and $\rho_{D}=\rho_{D-2}$.

Proof.

(i) $\Rightarrow$ (ii). This follows from Theorem 8.5.

(ii) $\Rightarrow$ (i). Assume $\theta^{\prime}=k-2$; the proof when $\theta^{\prime}=-(k-2)($ or $\theta= \pm(k-2))$ is similar. Furthermore, assume $c_{i}=i(1 \leqslant i \leqslant D-2)$ and $\sigma_{D}=\sigma_{D-2}$. Using (6) and induction, we find $\rho_{i}=(k-2 i) / k(0 \leqslant i \leqslant D-1)$. Now define scalars $\alpha=\sigma$ and $\beta=2-\rho$. Using this information and (6), we find (24) holds for $1 \leqslant i \leqslant D-2$. Using the fact that $\sigma_{D}=\sigma_{D-2}$ and (6), we find (24) holds for $i=D-1$. The result now follows from Theorem 6.6.

REMARK 8.7. Observe that the equation $\sigma_{D}=\sigma_{D-2}$ gives us a polynomial of degree $D$ in $\theta$, and that $\theta= \pm k$ is a root of this polynomial.

\section{The CASe $\Delta_{2} \neq 0$}

In this section we will classify the taut pairs of pseudo eigenvalues in the case where $\Delta_{2} \neq 0$. We will need the following definition.

DeFinition 9.1. With reference to Definition 6.1 , by the weights of the pair of pseudo eigenvalues $\theta, \theta^{\prime}$, we mean the complex scalars $\alpha, \beta$ given by the formulae in (25), (26). 
THEOREM 9.2. With reference to Definition 6.1 , assume that $\Delta_{2} \neq 0$. Furthermore, assume that $\theta, \theta^{\prime}$ is a taut pair, with $\theta, \theta^{\prime}$ nonzero. Let $\alpha, \beta$ denote the corresponding weights. Then

$$
\begin{array}{ll}
\frac{\sigma_{i+1}-\alpha \sigma_{i}}{\sigma \sigma_{i}-\sigma_{i-1}}=\frac{\beta \rho_{i}-\rho_{i-1}}{\rho \rho_{i}-\rho_{i-1}} & (1 \leqslant i \leqslant D-2), \\
\frac{\rho_{i+1}-\beta \rho_{i}}{\rho \rho_{i}-\rho_{i-1}}=\frac{\alpha \sigma_{i}-\sigma_{i-1}}{\sigma \sigma_{i}-\sigma_{i-1}} & (1 \leqslant i \leqslant D-2) .
\end{array}
$$

Observe the denominators in (59), (60) are nonzero by Corollaries 3.6, 7.5. Furthermore, if $\sigma_{D} \neq \sigma_{D-2}, \rho_{D} \neq \rho_{D-2}$, then (59), (60) also hold for $i=D-1$.

Proof. First, observe that for $1 \leqslant i \leqslant D-1$,

$$
\left(\sigma \sigma_{i}-\sigma_{i+1}\right)\left(\rho_{i-1}-\rho_{i+1}\right)=\left(\rho \rho_{i}-\rho_{i+1}\right)\left(\sigma_{i-1}-\sigma_{i+1}\right)
$$

since both sides equal $c_{i}\left(\rho_{i-1}-\rho_{i+1}\right)\left(\sigma_{i-1}-\sigma_{i+1}\right) / k$ in view of (9). Multiplying out (61) and cancelling terms, we obtain

$$
\sigma_{i+1} \rho_{i-1}-\sigma_{i-1} \rho_{i+1}=\sigma \sigma_{i}\left(\rho_{i-1}-\rho_{i+1}\right)-\rho \rho_{i}\left(\sigma_{i-1}-\sigma_{i+1}\right) .
$$

Multiplying (24) by $\sigma \sigma_{i}-\sigma_{i-1}$, multiplying (62) by $\sigma_{i+1}-\alpha \sigma_{i}$, and taking the difference between the two products, we find $\sigma_{i+1}-\sigma_{i-1}$ times

$$
\left(\sigma_{i+1}-\alpha \sigma_{i}\right)\left(\rho \rho_{i}-\rho_{i-1}\right)-\left(\beta \rho_{i}-\rho_{i-1}\right)\left(\sigma \sigma_{i}-\sigma_{i-1}\right)
$$

is zero. Observe $\sigma_{i+1}-\sigma_{i-1}$ is nonzero for $1 \leqslant i \leqslant D-2$ by Corollary 7.5, so (63) is zero. Line (59) follows. We obtain (60) by interchanging the roles of $\sigma_{j}, \rho_{j}(0 \leqslant j \leqslant$ $D-1)$ and the roles of $\alpha, \beta$ in the above argument.

LEMMA 9.3. With reference to Definition 6.1 , assume that $\Delta_{2} \neq 0$. Furthermore, assume that $\theta, \theta^{\prime}$ is a taut pair, with $\theta, \theta^{\prime}$ nonzero. Let $\alpha, \beta$ denote the corresponding weights. Then

$$
\frac{k}{b_{i}}=\frac{\alpha \sigma_{i}-\sigma_{i-1}}{\sigma \sigma_{i}-\sigma_{i-1}}+\frac{\beta \rho_{i}-\rho_{i-1}}{\rho \rho_{i}-\rho_{i-1}} \quad(1 \leqslant i \leqslant D-2) .
$$

Observe the denominators in (64) are nonzero by Corollaries 3.6, 7.5. Furthermore, if $\sigma_{D} \neq \sigma_{D-2}, \rho_{D} \neq \rho_{D-2}$, then (64) also holds for $i=D-1$.

Proof. Using (10), we find that for $1 \leqslant i \leqslant D-1$,

$$
\begin{aligned}
\frac{k}{b_{i}} & =\frac{\sigma_{i+1}-\sigma_{i-1}}{\sigma \sigma_{i}-\sigma_{i-1}} \\
& =\frac{\alpha \sigma_{i}-\sigma_{i-1}}{\sigma \sigma_{i}-\sigma_{i-1}}+\frac{\sigma_{i+1}-\alpha \sigma_{i}}{\sigma \sigma_{i}-\sigma_{i-1}} .
\end{aligned}
$$

Evaluating the fraction on the right in (66) using (59), we obtain (64).

With reference to Definition 6.1 , assume $\Delta_{2} \neq 0$. To classify the taut pairs of nonzero pseudo eigenvalues $\theta, \theta^{\prime}$, we will separate our results into two cases, depending on whether $\sigma_{D}=\sigma_{D-2}, \rho_{D}=\rho_{D-2}$. To see that there really are only two cases to consider, we need the following lemma.

LEMMA 9.4. With reference to Definition 6.1 , assume $\Delta_{2} \neq 0$ and that the pair $\theta, \theta^{\prime}$ is taut, where $\theta, \theta^{\prime}$ are nonzero. If $\sigma_{D}=\sigma_{D-2}$, then $\rho_{D}=\rho_{D-2}$.

Proof. Suppose to the contrary that $\sigma_{D}=\sigma_{D-2}$ but $\rho_{D} \neq \rho_{D-2}$. Observe $\alpha \neq \sigma$ by Corollary 7.5. Setting $i=D-1$ in (6), (24), we find $\sigma_{D}=\sigma \sigma_{D-1}$ and $\sigma_{D}=\alpha \sigma_{D-1}$. Thus $\sigma \sigma_{D-1}=\alpha \sigma_{D-1}$. Since $\alpha \neq \sigma$, we find $0=\sigma_{D-1}=\sigma_{D-2}=\sigma_{D}$. Using (6), we find $\sigma_{D-3}=0$, contradicting Corollary 7.5. 
THEOREM 9.5. Let $\Gamma$ denote a bipartite distance-regular graph with diameter $D \geqslant 5$ and valency $k \geqslant 3$. Let $\sigma_{0}, \sigma_{1}, \ldots, \sigma_{D}$ and $\rho_{0}, \rho_{1}, \ldots, \rho_{D}$ denote two sequences of complex numbers satisfying $\sigma_{D} \neq \sigma_{D-2}, \rho_{D} \neq \rho_{D-2}$. Abbreviate $\sigma=\sigma_{1}, \rho=\rho_{1}$. Let $\alpha, \beta$ denote complex scalars. Assume $\Delta_{2} \neq 0$ and $\sigma \neq \pm \rho$. Then the following (i), (ii) are equivalent.

(i) $\sigma_{0}, \sigma_{1}, \ldots, \sigma_{D}$ and $\rho_{0}, \rho_{1}, \ldots, \rho_{D}$ form a taut pair of pseudo cosine sequences where $\sigma, \rho \neq 0$, and where $\alpha, \beta$ are the weights of the corresponding pseudo eigenvalues.

(ii) $\sigma_{0}=1, \rho_{0}=1, \sigma \neq \alpha, \rho \neq \beta$, the polynomial in (16) has distinct roots (where $\left.\theta=k \sigma, \theta^{\prime}=k \rho\right)$, and

$$
\begin{aligned}
& \frac{\sigma_{i+1}-\alpha \sigma_{i}}{\sigma \sigma_{i}-\sigma_{i-1}}=\frac{\beta \rho_{i}-\rho_{i-1}}{\rho \rho_{i}-\rho_{i-1}} \quad(1 \leqslant i \leqslant D-1),(1 \leqslant i \leqslant D-1), \\
& \frac{\rho_{i+1}-\beta \rho_{i}}{\rho \rho_{i}-\rho_{i-1}}=\frac{\alpha \sigma_{i}-\sigma_{i-1}}{\sigma \sigma_{i}-\sigma_{i-1}} \quad(1 \leqslant i \leqslant D-1), \\
& \frac{k}{b_{i}}=\frac{\alpha \sigma_{i}-\sigma_{i-1}}{\sigma \sigma_{i}-\sigma_{i-1}}+\frac{\beta \rho_{i}-\rho_{i-1}}{\rho \rho_{i}-\rho_{i-1}} \quad(1 \leqslant .)
\end{aligned}
$$

and the denominators in (67)-(69) are nonzero.

Proof.

(i) $\Rightarrow$ (ii). We assume $\sigma_{0}, \sigma_{1}, \ldots, \sigma_{D}$ is a pseudo cosine sequence, so $\sigma_{0}=1$ by Corollary 3.5(ii). Similarly, $\rho_{0}=1$. Observe the polynomial in (16) has distinct roots by Theorem 6.6, and $\sigma \neq \alpha, \rho \neq \beta$ by Corollary 7.5. Equations (67), (68), (69) follow from Theorem 9.2 and Lemma 9.3.

(ii) $\Rightarrow$ (i). Replacing the rightmost fraction in (69) using (67) and simplifying, we obtain

$$
b_{i}\left(\sigma_{i+1}-\sigma_{i-1}\right)=k\left(\sigma \sigma_{i}-\sigma_{i-1}\right) \quad(1 \leqslant i \leqslant D-1) .
$$

We assume $\sigma_{0}=1$, so $\sigma_{0}, \sigma_{1}, \ldots, \sigma_{D}$ is a pseudo cosine sequence of $\Gamma$ by Corollary 3.5. Similarly, $\rho_{0}, \rho_{1}, \ldots, \rho_{D}$ is a pseudo cosine sequence of $\Gamma$. Observe the pseudo eigenvalues $\theta, \theta^{\prime}$ are nontrivial, since otherwise we would have a zero denominator in (67) at $i=1$. We show $\theta, \theta^{\prime}$ is a taut pair. To do this, we show (24) holds for $1 \leqslant i \leqslant D-1$. Fixing an integer $i(1 \leqslant i \leqslant D-1)$, we multiply the equations (67), (68) together and simplify to obtain

$$
\left(\sigma_{i+1}-\alpha \sigma_{i}\right)\left(\rho_{i+1}-\beta \rho_{i}\right)=\left(\beta \rho_{i}-\rho_{i-1}\right)\left(\alpha \sigma_{i}-\sigma_{i-1}\right) .
$$

Expanding (71), we routinely obtain (24). We have now shown (24) holds for $1 \leqslant i \leqslant$ $D-1$. Combining this with Theorem 6.6 , we find $\theta, \theta^{\prime}$ is a taut pair with weights $\alpha, \beta$. Finally, we note $\sigma, \rho$ are nonzero by Lemma 7.2 .

THEOREM 9.6. Let $\Gamma$ denote a bipartite distance-regular graph with diameter $D \geqslant 5$ and valency $k \geqslant 3$. Let $\sigma_{0}, \sigma_{1}, \ldots, \sigma_{D}$ and $\rho_{0}, \rho_{1}, \ldots, \rho_{D}$ denote two sequences of complex numbers satisfying $\sigma_{D}=\sigma_{D-2}, \rho_{D}=\rho_{D-2}$. Abbreviate $\sigma=\sigma_{1}, \rho=\rho_{1}$. Let $\alpha, \beta$ denote complex scalars. Assume $\Delta_{2} \neq 0$ and $\sigma \neq \pm \rho$. Then the following (i), (ii) are equivalent.

(i) $\sigma_{0}, \sigma_{1}, \ldots, \sigma_{D}$ and $\rho_{0}, \rho_{1}, \ldots, \rho_{D}$ form a taut pair of pseudo cosine sequences where $\sigma, \rho \neq 0$, and where $\alpha, \beta$ are the weights of the corresponding pseudo eigenvalues. 
(ii) $\sigma_{0}=1, \rho_{0}=1, \sigma \sigma_{D-1}=\sigma_{D}, \rho \rho_{D-1}=\rho_{D}, \sigma \neq \alpha, \rho \neq \beta$, the polynomial in (16) has distinct roots (where $\theta=k \sigma, \theta^{\prime}=k \rho$ ), and

$$
\begin{aligned}
& \frac{\sigma_{i+1}-\alpha \sigma_{i}}{\sigma \sigma_{i}-\sigma_{i-1}}=\frac{\beta \rho_{i}-\rho_{i-1}}{\rho \rho_{i}-\rho_{i-1}} \quad(1 \leqslant i \leqslant D-2),(1 \leqslant i \leqslant D-2), \\
& \frac{\rho_{i+1}-\beta \rho_{i}}{\rho \rho_{i}-\rho_{i-1}}=\frac{\alpha \sigma_{i}-\sigma_{i-1}}{\sigma \sigma_{i}-\sigma_{i-1}} \quad(1 \leqslant i \leqslant D-2), \\
& \frac{k}{b_{i}}=\frac{\alpha \sigma_{i}-\sigma_{i-1}}{\sigma \sigma_{i}-\sigma_{i-1}}+\frac{\beta \rho_{i}-\rho_{i-1}}{\rho \rho_{i}-\rho_{i-1}} \quad(1 \leqslant
\end{aligned}
$$

and the denominators in (72)-(74) are nonzero.

Furthermore, suppose (i), (ii) hold above. Then $b_{D-2}=b_{D-1}=c_{2}=1$.

Proof. Similar to the proof of Theorem 9.5. To finish the proof of (i) $\Rightarrow$ (ii), we note that the equation $\sigma \sigma_{D-1}=\sigma_{D}$ follows from (9) and our assumption that $\sigma_{D}=\sigma_{D-2}$. Similarly, $\rho \rho_{D-1}=\rho_{D}$.

To finish the proof of (ii) $\Rightarrow$ (i), we note that (24) trivially holds for $i=D-1$, since we assume $\sigma_{D}=\sigma_{D-2}, \rho_{D}=\rho_{D-2}$. The final statement follows from Lemma 7.4.

With reference to Definition 6.1 , assume $\Delta_{2} \neq 0$. In the following theorem, we show that $\Gamma$ has essentially at most one taut pair of nonzero pseudo eigenvalues $\theta, \theta^{\prime}$, and that any other taut pairs are obtained by taking plus or minus of these pseudo eigenvalues.

THEOREM 9.7. Let $\Gamma$ denote a bipartite distance-regular graph with diameter $D \geqslant 5$ and valency $k \geqslant 3$. Assume that $\Delta_{2} \neq 0$. Let $\theta, \theta^{\prime}, \psi, \psi^{\prime}$ denote nonzero nontrivial pseudo eigenvalues. Suppose the pairs $\theta, \theta^{\prime}$ and $\psi, \psi^{\prime}$ are both taut. Then $\left\{\theta^{2}, \theta^{\prime 2}\right\}=$ $\left\{\psi^{2}, \psi^{\prime 2}\right\}$.

Proof. Immediate from Theorem 4.10 and Lemma 4.11.

\section{REFERENCES}

[1] Eiichi Bannai and Tatsuro Ito, Algebraic combinatorics I, association schemes, Mathematics lecture note series, vol. 58, Benjamin/Cummings Publishing Company, Inc, 1984.

[2] Andries E. Brouwer, Arjeh M. Cohen, and Arnold Neumaier, Distance-regular graphs, A Series of Modern Surveys in Mathematics, Springer-Verlag, 1989.

[3] Brian Curtin, 2-homogeneous bipartite distance-regular graphs, Discrete Mathematics 187 (1998), no. 1-3, 39-70.

[4] _ Almost 2-homogeneous bipartite distance-regular graphs, European Journal of Combinatorics 21 (2000), no. 7, 865-876.

[5] Aleksandar Jurišić and Paul Terwilliger, Pseudo 1-homogeneous distance-regular graphs, Journal of Algebraic Combinatorics 28 (2008), no. 4, 509-529.

[6] Michael S Lang, Pseudo primitive idempotents and almost 2-homogeneous bipartite distanceregular graphs, European Journal of Combinatorics 29 (2008), no. 1, 35-44.

[7] _ Bipartite distance-regular graphs: the Q-polynomial property and pseudo primitive idempotents, Discrete Mathematics 331 (2014), 27-35.

[8] Mark S MacLean, Taut distance-regular graphs of odd diameter, Journal of Algebraic Combinatorics 17 (2003), no. 2, 125-147.

[9] $\ldots$, The local eigenvalues of a bipartite distance-regular graph, European Journal of Combinatorics 45 (2015), 115-123.

[10] Mark S MacLean, Štefko Miklavič, and Safet Penjić, On the Terwilliger algebra of bipartite distance-regular graphs with $\Delta_{2}=0$ and $c_{2}=1$, Linear Algebra and its Applications 496 (2016), 307-330.

[11] Mark S MacLean and Paul Terwilliger, Taut distance-regular graphs and the subconstituent algebra, Discrete mathematics 306 (2006), no. 15, 1694-1721.

[12] Arlene A Pascasio and Paul Terwilliger, The pseudo-cosine sequences of a distance-regular graph, Linear algebra and its applications 419 (2006), no. 2-3, 532-555. 
[13] Safet Penjić, On the Terwilliger algebra of bipartite distance-regular graphs with $\Delta_{2}=0$ and $c_{2}=2$, Discrete Mathematics 340 (2017), no. 3, 452-466.

[14] Paul Terwilliger and Chih-wen Weng, Distance-regular graphs, pseudo primitive idempotents, and the Terwilliger algebra, European Journal of Combinatorics 25 (2004), no. 2, 287-298.

Mark S. MacLean, Mathematics Department, Seattle University, 901 Twelfth Avenue, Seattle WA 98122-1090, USA

E-mail : macleanm@seattleu.edu

Štefko Miklavič, University of Primorska, Andrej Marušič Institute, Muzejski trg 2, 6000 Koper, Slovenia

E-mail : stefko.miklavic@upr.si 